\title{
Exponentiation of Multiparticle Amplitudes in Scalar Theories
}

\author{
M. V. Libanov, V. A. Rubakov, D. T. Son and S. V. Troitsky \\ Institute for Nuclear Research of the Russian Academy of Sciences, \\ 60th October Anniversary Prospect 7a, Moscow 117312 Russia.
}

(July 1994)

\begin{abstract}
It is argued that the amplitudes of the production of $n$ soft scalar particles by one or a few energetic ones in theories like $\lambda \varphi^{4}$ has the exponential form, $A_{n} \propto \sqrt{n !} \exp \left[\frac{1}{\lambda} F(\lambda n, \epsilon)\right]$, in the regime $\lambda \rightarrow 0, \lambda n=$ fixed, $\epsilon=$ fixed, where $\epsilon$ is the typical kinetic energy of outgoing particles. Existing results support this conjecture. Several new analytical and numerical results in favor of the exponential behavior of multiparticle amplitudes are presented.
\end{abstract}




\section{INTRODUCTION}

Two classes of processes in weakly coupled theories with multiparticle final states attract considerable interest. One of them is the instanton-like transitions induced by collisions of highly energetic particles (which violate $(\mathrm{B}+\mathrm{L})$ in the standard electroweak theory) [1,2]; there the typical number of final particles is expected to be of order $1 / \alpha$ at energies $E \propto$ $m / \alpha$, where $m$ and $\alpha$ are mass of a relevant particle and coupling constant, respectively ( $m=m_{W}, \alpha=\alpha_{W}$ in the electroweak theory; for reviews see refs. [3, 1 ). The other is the "perturbative" creation of $n \propto 1 / \lambda$ soft particles by one or a few energetic (virtual or real) ones in scalar theories like $\lambda \varphi^{4}$ [6,5, 7]. In both cases the amplitudes calculated to the leading order of the perturbation theory about either the instanton or vacuum are unacceptably large, corrections to them exceed the leading order results and the conventional perturbation theory breaks down.

In the case of the instanton-like processes, there exist strong arguments showing that the total probability has the exponential form [8 11]

$$
\sigma \propto \mathrm{e}^{\frac{1}{\alpha} F\left(E / E_{0}\right)}
$$

where $E_{0} \propto \frac{m}{\alpha}$ and the pre-exponential factor is at most a power of $\alpha$ at $E \sim E_{0}$. Eq.(1.1) suggests that there may exist a semiclassical-type technique for calculating the exponent $F\left(E / E_{0}\right)$; several ideas have been put forward in this direction 12 17.

In this paper we conjecture that the behavior similar to eq.(1.1) is characteristic to multiparticle amplitudes in scalar theories. Namely, consider a theory of one scalar field with the lagrangian

$$
L=\frac{1}{2}\left(\partial_{\mu} \varphi\right)^{2}-\frac{m^{2}}{2} \varphi^{2}-\frac{\lambda}{4} \varphi^{4}
$$

(we will set the mass of the boson equal to one wherever possible, the powers of $m$ can be reconstructed on dimensional grounds). The processes we are interested in are creation of $n$ scalar quanta with typical kinetic energy $\epsilon$ by one or a few initial (virtual or real) particles, as illustrated in fig.1. We conjecture that in the limit 


$$
\lambda \rightarrow 0, \quad \lambda n=\text { fixed }, \quad \epsilon=\text { fixed }
$$

the amplitudes of these processes have the form

$$
A_{n} \propto \sqrt{n !} e^{\frac{1}{\lambda} F(\lambda n, \epsilon)}
$$

where the pre-exponential factor is at most some power of $\lambda$. This behavior is expected to be inherent in both unbroken theory $\left(m^{2}>0\right)$ and broken theory $\left(m^{2}<0\right)$, as well as in extensions of the model (1.2), the function $F$ being model dependent. If there are other parameters in a model, like the number of scalar fields in $O(N)$ theory, they are all assumed to be fixed in the limit (1.3).

At the moment, we are unable to prove eq.(1.4). Instead, in this paper we present a series of consistency checks which will be summarized in the rest of this section. Before doing so, let us make a few comments concerning eq.(1.4).

First, eq.(1.4) implies that the cross section of the creation of $n$ soft scalar particles,

$$
\sigma_{n} \propto \frac{\left|A_{n}\right|^{2}}{n !} \times(\text { phase space })
$$

is exponential in the regime (1.3),

$$
\sigma_{n} \propto \mathrm{e}^{\frac{1}{\lambda} G(n \lambda, \epsilon)}
$$

Eq.(1.5) is completely analogous to eq.(1.1) characteristic to the instanton-like transitions. Whether the cross section (1.5) is small at all $n$ or becomes large at $n \propto 1 / \lambda$ is determined by the actual behavior of the function $F(n \lambda, \epsilon)$ on which we have almost nothing to say in this paper.

Second, there exist arguments based on the ordinary perturbation theory at low momenta (say, for the scalar propagator) and unitarity, which favor the exponential suppression of the $f e w \rightarrow n$ cross sections at $n \propto 1 / \lambda$ [18]. Eqs.(11.4) and (1.5) are consistent with this expectation provided that $F(n \lambda, \epsilon)$ is always negative and decreases with $\epsilon$ rapidly enough. We think, however, that the actual computation of $F(n \lambda, \epsilon)$ is necessary to settle the issue. 
Finally, the obvious generalization of eq.(1.4) to the processes $n_{1} \rightarrow n_{2}$ is that the connected amplitude has the following form,

$$
A_{n_{1} \rightarrow n_{2}} \propto \sqrt{n_{1} !} \sqrt{n_{2} !} \mathrm{e}^{\frac{1}{\lambda} \tilde{F}\left(n_{1} \lambda, n_{2} \lambda, \epsilon\right)}
$$

which we conjecture to hold in the regime

$$
\lambda \rightarrow 0, \quad \lambda n_{1}=\text { fixed, } \quad \lambda n_{2}=\text { fixed }, \quad \epsilon=\text { fixed }
$$

Eq.(1.6) has a direct analog in the instanton-like case [12 14]; in this paper we will present the arguments in favor of eq.(1.6) in the scalar theory.

Let us now summarize the consistency checks for the conjecture (1.4).

i) Tree amplitudes at threshold in $\varphi^{4}$ theory. At $\epsilon=0$ the amplitudes $1 \rightarrow n$ have been calculated at the tree level in refs. [0,19,20],

$$
\begin{array}{ll}
A_{n}^{\text {tree }}=n !\left(\frac{\lambda}{8}\right)^{\frac{n-1}{2}} & (\text { unbroken theory) } \\
A_{n}^{\text {tree }}=n !\left(\frac{\lambda}{2}\right)^{\frac{n-1}{2}} & \text { (broken theory) }
\end{array}
$$

In the limit (1.3) these amplitudes indeed have the form (1.4) with

$$
\begin{array}{cl}
F^{\text {tree }}=\frac{(\lambda n)}{2} \ln \frac{\lambda n}{8}-\frac{\lambda n}{2} & \text { (unbroken theory) } \\
F^{\text {tree }}=\frac{(\lambda n)}{2} \ln \frac{\lambda n}{2}-\frac{\lambda n}{2} & \text { (broken theory) }
\end{array}
$$

ii) Tree amplitudes at threshold in an extension of $\varphi^{4}$ theory. The model of two scalar fields $\varphi_{1}$ and $\varphi_{2}$ with unequal masses and $O(2)$ symmetric interaction, described by the lagrangian

$$
L=\frac{1}{2}\left(\partial_{\mu} \varphi_{1}\right)^{2}+\frac{1}{2}\left(\partial_{\mu} \varphi_{2}\right)^{2}-\frac{m_{1}^{2}}{2} \varphi_{1}^{2}-\frac{m_{2}^{2}}{2} \varphi_{2}^{2}-\frac{\lambda}{4}\left(\varphi_{1}^{2}+\varphi_{2}^{2}\right)^{2}
$$

has been studied in ref. [21]. The tree amplitudes at the threshold for the processes $1 \rightarrow$ $\left(n_{1}, n_{2}\right)$ have been calculated exactly. At $n_{1} \propto 1 / \lambda, n_{2} \propto 1 / \lambda$ they have the form 


$$
A_{n_{1} \rightarrow n_{2}}^{\text {tree }} \propto \sqrt{n_{1} !} \sqrt{n_{2} !} e^{\frac{1}{\lambda} F^{\text {tree }}\left(n_{1} \lambda, n_{2} \lambda\right)}
$$

The exponent is particularly simple when $n_{1} / n_{2}=1+O(1 / \lambda)$ where (we assume for definiteness that the incoming virtual boson is of the first type, $\varphi_{1}$ )

$$
F(\lambda n)=n \lambda\left(\ln \frac{n \lambda\left(\sqrt{m_{1}}+\sqrt{m_{2}}\right)^{2}}{8\left(m_{1}+m_{2}\right)}-1\right)
$$

(unbroken theory),

$$
F(\lambda n)=n \lambda\left(\ln \frac{n \lambda\left(\sqrt{m_{1}}+\sqrt{2 m_{2}}\right)^{2}}{2\left(m_{1}+2 m_{2}\right)}-1\right)
$$

(broken theory, $m_{1}^{2}<0$ or $m_{2}^{2}<0$ ).

Eq.(1.10) is an obvious generalization of eq.(1.4) for the theory (1.9).

iii) Energy dependence of the tree amplitudes near threshold in unbroken $\varphi^{4}$

theory. At the tree level, the dependence of the $1 \rightarrow n$ amplitudes on $\lambda$ is known explicitly,

$$
A_{n}^{\text {tree }} \propto \lambda^{\frac{n}{2}}
$$

At small $\epsilon$, eqs. (1.4) and (1.11) are consistent with each other only if

$$
A_{n}^{\text {tree }}(\epsilon)=A_{n}^{\text {tree }}(\epsilon=0) \mathrm{e}^{A n\left[\epsilon+O\left(\epsilon^{2}\right)\right]}
$$

where $A$ is some constant. Since $n \epsilon=E$, the total c.m. kinetic energy, we expect the tree amplitudes of the creation of $n$ non-relativistic bosons to have the following form,

$$
A_{n}^{\text {tree }}(E)=A_{n}^{\text {tree }}(E=0) \mathrm{e}^{(A E+\ldots)}
$$

where dots denote the terms suppressed by $E / n$. We calculate the leading energy correction in $\varphi^{4}$-theory in sect.II of this paper and show that it indeed has the form (1.12) with

$$
A=-\frac{5}{6}
$$

iv) Exponentiation of the loop corrections at threshold in the leading order in $(n \lambda)$. The one loop correction to the $1 \rightarrow n$ amplitude at threshold in $\varphi^{4}$ theory has been calculated in refs. 22 24. The result is 


$$
A_{n}^{1 \text {-loop }}=A_{n}^{\text {tree }} \times\left(B \lambda n^{2}+O(\lambda n)\right)
$$

where the (complex) constant $B$ depends on the number of dimensions. In $(3+1)$ dimensions one has

$$
\begin{gathered}
B=-\frac{1}{64 \pi^{2}}(\ln (7+4 \sqrt{3})-i \pi) \quad \text { (unbroken theory) } \\
B=\frac{\sqrt{3}}{8 \pi} \quad \text { (broken theory) }
\end{gathered}
$$

At higher loops one expects higher powers of $n$ along with higher powers of $\lambda$. We show in sect.III of this paper that in the $k$-th loop, the leading- $n$ correction has the form

$$
A_{n}^{\text {tree }} \cdot \frac{B^{k}}{k !} \cdot\left(\lambda n^{2}\right)^{k}(1+O(1 / n))
$$

with exactly the same constant $B$ as in eqs. (1.14), (1.15). This means that these leading order corrections, being summed over all loops, exponentiate,

$$
A_{n}=A_{n}^{\text {tree }} \mathrm{e}^{B \lambda n^{2}} \times \cdots
$$

which is consistent with eq.(1.4) with

$$
F(\lambda n)=F^{\text {tree }}(\lambda n)+B \cdot(\lambda n)^{2}+\cdots
$$

The non-leading terms in eqs. (1.13) and (1.16) correspond, presumably, to terms of order $(\lambda n)^{3},(\lambda n)^{4}$, etc., in $F(\lambda n)$ and to the contributions to the pre-exponential factor in eq.(1.4).

In analogy to the instanton-like processes, eqs. (1.7), (1.8) and (1.18) imply that the tree result is actually a good approximation to $F$ at small $\lambda n$, in spite of the fact that the one loop correction (1.13) is greater than the tree amplitude (the point is of course that even small correction to $F$ leads to the large factor in the amplitude, see eq. (11.17)). On the other hand, at $\lambda n \sim 1$ the series (1.18) blows up at $\lambda n \sim 1$, and truly non-perturbative techniques is needed for calculating $F(\lambda n)$.

v) Amplitudes at threshold in $O(N)$ theory at large $N$. The amplitudes of $1 \rightarrow n$ processes at threshold in the $O(N)$ symmetric theory of $N$ scalar fields have been calculated in ref. [25] to all loops in the regime 


$$
\lambda \rightarrow 0, \quad \lambda N=\text { fixed }, \quad n=\text { fixed }
$$

They are equal to

$$
A_{n}^{N}=\left(\frac{\lambda}{8(1+c \lambda N)}\right)^{n / 2} \cdot n !
$$

where the coefficient $c$ depends on the renormalization scheme. The regime (1.19) is different from the regime

$$
\lambda \rightarrow 0, \quad \lambda n=\text { fixed }
$$

with all other parameters (including $N$ ) fixed, which is considered throughout this paper. The two regimes match only in the region $n \lambda \ll 1, N \lambda \ll 1$ where eq.(1.20) is consistent with eq.(1.4) with $N$-dependent pre-exponential factor

$$
A^{N}=\mathrm{e}^{\text {const } \cdot(\lambda n) N} \cdot A^{\text {tree }, N=1}
$$

So, there is no contradiction between the large $-N$ results and our conjecture.

vi) Infrared behavior of the amplitudes in $\varphi^{4}$ theory in $(2+1)$ dimensions near threshold. Loop contributions to the amplitudes exactly at the threshold are infrared divergent in the $\varphi^{4}$ theory in $(2+1)$ dimensions. At small but non-zero $\epsilon$, the terms of order $(\lambda \ln \epsilon)^{k}$ appear in $k$-th loop. These terms can be summed up by the renormalization group technique 26] with the result

$$
\begin{array}{cc}
A_{n}(\epsilon)=A_{n}^{\text {tree }}\left(1-\frac{3 \lambda}{16 \pi} \ln \epsilon\right)^{-\frac{n(n-1)}{2}} & \text { (unbroken theory) } \\
A_{n}(\epsilon)=A_{n}^{\text {tree }}\left(1+\frac{3 \lambda}{4 \pi} \ln \epsilon\right)^{-\frac{n(n-1)}{2}} & \text { (broken theory) }
\end{array}
$$

which is valid in the leading logarithmic regime

$$
\lambda \rightarrow 0, \quad \epsilon \rightarrow 0, \quad \lambda \ln \epsilon=\text { fixed }, \quad n=\text { fixed }
$$

This regime matches with one considered in this paper, eq.(1.3), at 


$$
\epsilon \ll 1, \quad \lambda \ln \epsilon \ll 1, \quad \lambda n \ll 1
$$

where eqs. (1.21) and (1.22) agree with eq.(1.4) with

$$
\begin{gathered}
F(\epsilon)=F^{\text {tree }}+\frac{3}{32 \pi}(\lambda n)^{2} \ln \epsilon \quad \text { (unbroken theory) } \\
F(\epsilon)=F^{\text {tree }}-\frac{3}{8 \pi}(\lambda n)^{2} \ln \epsilon \quad \text { (broken theory) }
\end{gathered}
$$

Similar results hold for $O(N)$ extension of the $\varphi^{4}$ theory. In the unbroken case one finds in the limit (1.23) and fixed $N$

$$
F(\epsilon)=F_{\epsilon=0}^{\text {tree }}+\frac{3(\lambda n)^{2}}{32 \pi} \ln \epsilon
$$

We present the leading logarithm calculations of the $1 \rightarrow n$ amplitudes in $(2+1)$ dimensions in sect.TT of this paper for completeness.

vii) Finally, the conjecture (1.6) on the behavior of the connected amplitudes $n_{1} \rightarrow n_{2}$ can be tested at the tree level. The dependence of the amplitudes on $\lambda$ follows from counting the vertices in the tree graphs,

$$
A_{n_{1} \rightarrow n_{2}}^{\text {tree }} \propto \lambda^{\frac{n_{1}+n_{2}}{2}}
$$

so one expects from eq.(1.6) the following functional form of the tree amplitudes at rest at large $n_{1}$ and $n_{2}$,

$$
A_{n_{1} \rightarrow n_{2}}=n_{1} ! n_{2} ! \lambda^{\frac{n_{1}+n_{2}}{2}} \mathrm{e}^{\left(n_{1}+n_{2}\right) \Phi\left(n_{2} / n_{1}\right)}
$$

where $\Phi\left(n_{2} / n_{1}\right)$ is some unknown function. We present numerical results confirming the scaling behavior, eq.(1.24), in sect. प of this paper, where heuristic arguments in favor of the conjecture (1.6) are also given.

The rest of this paper is devoted to the evaluation of the new results entering the above arguments: energy dependence of the tree $1 \rightarrow n$ amplitudes near the threshold (sect.[I), leading $-n$ corrections to $1 \rightarrow n$ to all loops at the threshold (sect.[II), leading-log infrared behavior of $1 \rightarrow n$ amplitudes to all loops in (2+1) dimensions (sect. IV]), and $n_{1} \rightarrow n_{2}$ tree 
amplitudes at rest at large $n_{1}, n_{2}$ and fixed $n_{2} / n_{1}$ (sect. $\nabla$ ). Although these new results may be of interest by themselves, we think their main value is to support our conjectures, eqs.(1.4) and (1.6).

Sect.VI contains concluding remarks.

\section{ENERGY DEPENDENCE OF MULTIPARTICLE TREE AMPLITUDES AROUND THRESHOLD}

Our first consistency check of the exponentiation hypothesis is the calculation of the multiparticle tree amplitudes when final particles are not at rest, but have finite kinetic energies. We will be interested in the amplitude of the production of $n$ final particles with momenta $p_{i}$ from an initial virtual particle in the $\varphi^{4}$ theory without symmetry breaking. In what follows we consider the case of non-relativistic final particles, $p_{i} \ll 1$. Denote the total kinetic energy of final particles in the center-of-mass frame by $E$, in this frame $\left(\sum p_{i}=0\right)$ one has $E=\frac{1}{2} \sum p_{i}^{2}$ (we set the mass of scalar bosons equal to 1 ). More generally, one has the following formula for $E$ in arbitrary frame,

$$
\begin{gathered}
E=\frac{1}{2} \sum_{i=1}^{n} p_{i}^{2}-\frac{1}{2 n}\left(\sum_{i=1}^{n} p_{i}\right)^{2} \\
=\frac{n-1}{2 n} \sum_{i=1}^{n} p_{i}^{2}-\frac{1}{n} \sum_{i \neq j}^{n} p_{i} p_{j}
\end{gathered}
$$

The case of threshold production corresponds to $E=0$. In this section we show that at large $n$ and $E \ll n$, the tree amplitude has the following form

$$
A_{n}\left(p_{1}, \ldots, p_{n}\right)=n !\left(\frac{\lambda}{8}\right)^{\frac{n-1}{2}} \mathrm{e}^{-\frac{5}{6} E}
$$

As the first step for establishing this formula, we consider the region of very small $E, E \ll 1$, where the amplitude can be calculated for arbitrary $n$, not necessarily large. 


\section{A. Amplitude at $E \ll 1$ and arbitrary $n$.}

If the momenta of final particles $p_{i}$ are small, we can expand the tree amplitude $A_{n}\left(p_{i}\right)$ in powers of $p_{i}$. The zeroth term is the threshold amplitude $A_{n}$. Since the amplitude is $P$ invariant, $A\left(p_{i}\right)=A\left(-p_{i}\right)$, the first energy correction to the threshold amplitude is quadratic in $p_{i}$. The Galilean invariance and the symmetry of the amplitude under permutations of $p_{i}$ ensures that this quadratic correction is proportional to $E$. So, one writes

$$
A_{n}\left(p_{1}, \ldots, p_{n}\right)=A_{n}+\alpha_{n} E+O\left(E^{2}\right)
$$

where $\alpha_{n}$ is some constant that depends on $n$. The purpose of this subsection is to calculate $\alpha_{n}$.

The tree amplitude $A\left(p_{1}, \ldots, p_{n}\right)$ satisfies the following recurrence relation (see fig.2, $\mathrm{cf}$. [7.20 ,

$$
\begin{gathered}
\left((n+E)^{2}-\left(\sum_{i=1}^{n} p_{i}\right)^{2}-1\right) A_{n}\left(p_{1}, \ldots, p_{n}\right)= \\
\lambda \sum_{n_{1}, n_{2}, n_{3}} \sum_{\mathcal{P}} A_{n_{1}}\left(p_{1}^{(1)}, \ldots, p_{n_{1}}^{(1)}\right) A_{n_{2}}\left(p_{1}^{(2)}, \ldots, p_{n_{2}}^{(2)}\right) A_{n_{3}}\left(p_{1}^{(3)}, \ldots, p_{n_{3}}^{(3)}\right)
\end{gathered}
$$

where the sum is taken over all $n_{1}, n_{2}, n_{3}$ satisfying the relation $n_{1}+n_{2}+n_{3}=n$ and over all permutations $\mathcal{P}$ of momenta $p_{i}$. In fact, the sum runs over odd values of $n_{1}, n_{2}, n_{3}$ only, since in the theory without symmetry breaking the $1 \rightarrow n$ amplitude vanishes at even $n$. For convenience we will work in the center-of-mass frame where $\sum_{i=1}^{n} p_{i}=0$. Hereafter we include the initial propagator into the amplitude $A_{n}\left(p_{1}, \ldots, p_{n}\right)$.

Let us expand both left and right hand sides of eq.(2.3) in powers of $E$. At the zeroth order in energy, one obtains the recurrence relation for the threshold amplitude $A_{n}$,

$$
\left(n^{2}-1\right) A_{n}=\lambda \sum_{n_{1}, n_{2}, n_{3}} \frac{n !}{n_{1} ! n_{2} ! n_{3} !} A_{n_{1}} A_{n_{2}} A_{n_{3}}
$$

while in the first order in energy $E$ one has

$$
\left(n^{2}-1\right) \alpha_{n}+2 n A_{n}=3 \lambda \sum_{n_{1}, n_{2}, n_{3} ; n_{1}>1} \frac{n !}{n_{1} ! n_{2} ! n_{3} !} \frac{n_{1}-1}{n-1} \alpha_{n_{1}} A_{n_{2}} A_{n_{3}}
$$


By introducing the generating functions

$$
\begin{array}{r}
A(\tau)=\sum_{n=1}^{\infty} \frac{A_{n}}{n !} \mathrm{e}^{n \tau} \\
\alpha(\tau)=\sum_{n=3}^{\infty} \frac{\alpha_{n}}{n !}(n-1) \mathrm{e}^{n \tau}
\end{array}
$$

the recurrence relations, eqs.(2.4), (2.5), can be rewritten in the form of differential equations for $A(\tau)$ and $\alpha(\tau)$,

$$
\begin{gathered}
\left(\partial_{\tau}^{2}-1\right) A(\tau)=\lambda A^{3}(\tau) \\
\left(\partial_{\tau}^{2}-1\right) \alpha(\tau)+2 \partial_{\tau}^{2} A(\tau)-2 \partial_{\tau} A(\tau)=3 \lambda \alpha(\tau) A^{2}(\tau)
\end{gathered}
$$

In particular, the equation for $A(\tau)$ coincides with the field equation for spatially homogeneous configurations. Let us discuss the boundary conditions for $A(\tau)$ and $\alpha(\tau)$. The $1 \rightarrow 1$ amplitude at arbitrary momentum is $A_{1}=1$. So, the boundary conditions are

$$
\begin{aligned}
& A(\tau)=\mathrm{e}^{\tau}+O\left(\mathrm{e}^{3 \tau}\right) \\
& \alpha(\tau)=O\left(\mathrm{e}^{3 \tau}\right)
\end{aligned}
$$

in the limit $\tau \rightarrow-\infty$. The solution to eqs.(2.7) with boundary conditions (2.8) is

$$
\begin{gathered}
A(\tau)=\frac{\mathrm{e}^{\tau}}{1-\frac{\lambda}{8} \mathrm{e}^{2 \tau}} \\
\alpha(\tau)=\frac{1}{6}\left[\frac{\mathrm{e}^{\tau}}{1-\frac{\lambda}{8} \mathrm{e}^{2 \tau}}-\mathrm{e}^{\tau}-\frac{5 \lambda}{4} \frac{\mathrm{e}^{3 \tau}}{\left(1-\frac{\lambda}{8} \mathrm{e}^{2 \tau}\right)^{2}}\right]
\end{gathered}
$$

Expanding (2.9) in powers of $\mathrm{e}^{\tau}$, we obtain $A_{n}$ and $\alpha_{n}$,

$$
\begin{gathered}
A_{n}=n !\left(\frac{\lambda}{8}\right)^{\frac{n-1}{2}}, \quad n \text { odd } \\
\alpha_{n}=-n !\left(\frac{\lambda}{8}\right)^{\frac{n-1}{2}} \frac{1}{6}\left(5-\frac{1}{n-1}\right), \quad n \text { odd }, n \geq 3
\end{gathered}
$$


Thus, up to the first order in $E$, the tree amplitude is

$$
A\left(p_{1}, \ldots, p_{n}\right)=n !\left(\frac{\lambda}{8}\right)^{\frac{n-1}{2}}\left[1-\left(\frac{5}{6}-\frac{1}{6(n-1)}\right) E\right]
$$

From eq. (2.10) one sees that the expansion in $E$ is a good approximation only when $E \ll 1$, i.e when the total kinetic energy of final particles is much smaller than the energy required for creating an additional scalar boson, and the energy per a final particle is much smaller than $1 / n$. However, at large $n$, one can calculate the amplitude in a larger range of $E$. In the next subsection we will obtain the expression for the tree amplitude at $E \ll n$, which covers the whole non-relativistic final phase space.

\section{B. Non-relativistic tree amplitudes at large $n$}

For reasons explained in Introduction, we expect that at large $n$ and $E \ll n$ the amplitude has the following form,

$$
A\left(p_{1}, \ldots, p_{n}\right)=A_{n} \mathrm{e}^{A E}=n !\left(\frac{\lambda}{8}\right)^{\frac{n-1}{2}} \mathrm{e}^{A E}
$$

where $A$ is some constant. Let us verify that at large $n$ the Ansatz (2.11) satisfies the recurrence relation (2.3) with the accuracy of $E / n \ll 1$.

Substituting (2.11) to (2.3), one has on the left hand side of eq.(2.3),

$$
\left((n+E)^{2}-1\right) A\left(p_{i}\right)=n^{2} A_{n} \mathrm{e}^{A E}(1+O(E / n))
$$

Consider now the right hand side of eq.(2.3). Taking into account eq.(2.1), one writes

$$
\begin{aligned}
& \lambda \sum_{n_{1}, n_{2}, n_{3}} \sum_{\mathcal{P}} A_{n_{1}} A_{n_{2}} A_{n_{3}} \exp \left(\frac{n_{1}-1}{2 n_{1}} A \sum_{i=1}^{n_{1}} p_{i}^{(1)^{2}}-\frac{A}{n_{1}} \sum_{i \neq j}^{n_{1}} p_{i}^{(1)} p_{j}^{(1)}+((1) \rightarrow(2),(3))\right) \\
= & 8 A_{n} \mathrm{e}^{A E} \sum_{n_{1}, n_{2}, n_{3}} \frac{n_{1} ! n_{2} ! n_{3} !}{n !} \sum_{\mathcal{P}} \exp \left(-\frac{A}{2 n_{1}} \sum p_{1}^{(1)^{2}}-\frac{A}{n_{1}} \sum p_{i}^{(1)} p_{j}^{(1)}+((1) \rightarrow(2),(3))\right)
\end{aligned}
$$

Let us expand the exponential function in powers of momenta. The first term is equal to one. If one retains only this term, the sum is 


$$
\sum_{n_{1}, n_{2}, n_{3}} \sum_{\mathcal{P}} \frac{n_{1} ! n_{2} ! n_{3} !}{n !}=\frac{n^{2}-1}{8}
$$

(note that the sum runs over odd values of $\left.n_{1}, n_{2}, n_{3}\right)$. One finds that the expressions (2.12) and (2.13) coincide with the accuracy of $1 / n$, i.e., the recurrence relation (2.3) is satisfied with this accuracy. So, to show that eq.(2.3) is satisfied by our Ansatz, one has to check that the contributions from higher terms in the Taylor expansion of the exponent to the sum in eq. 2.13) are suppressed.

Let us check that the second term in the Taylor series of the exponent indeed gives a small contribution to the right hand side of eq.(2.3). To evaluate the sum that comes from this term,

$$
\sum_{n_{1}, n_{2}, n_{3}} \frac{n_{1} ! n_{2} ! n_{3} !}{n !} \sum_{\mathcal{P}}\left(\frac{A}{2 n_{1}} \sum p_{1}^{(1)^{2}}+\frac{A}{n_{1}} \sum p_{i}^{(1)} p_{j}^{(1)}+((1) \rightarrow(2),(3))\right)
$$

one makes use of the following combinatoric relations,

$$
\begin{gathered}
\frac{n_{1} ! n_{2} ! n_{3} !}{n !} \sum_{\mathcal{P}} \sum_{i=1}^{n_{1}} p_{i}^{(1)^{2}}=\frac{n_{1}}{n} \sum_{i=1}^{n} p_{i}^{2} \\
\frac{n_{1} ! n_{2} ! n_{3} !}{n !} \sum_{\mathcal{P}} \sum_{i \neq j}^{n_{1}} p_{i}^{(1)} p_{j}^{(1)}=\frac{n_{1}\left(n_{1}-1\right)}{n(n-1)} \sum_{i \neq j}^{n} p_{i} p_{j}
\end{gathered}
$$

The contribution from the second term in the Taylor series to (2.13) is then

$$
\begin{gathered}
-8 A_{n} \mathrm{e}^{A E} \sum_{n_{1}, n_{2}, n_{3}}\left(\frac{3 A}{2 n} \sum_{i=1}^{n} p_{i}^{2}+\frac{n-3}{n(n-1)} A \sum_{i \neq j}^{n} p_{i} p_{j}\right) \\
=-16 A_{n} \mathrm{e}^{A E}(n+1) A E
\end{gathered}
$$

where we have made use of the relations $\sum p_{i}^{2}=2 E, \sum p_{i} p_{j}=-E$. It is clear that at large $n$, the expression (2.14) is suppressed by a factor of $E / n$ as compared to the leading term, eq. (2.12). So, in the case $E \ll n$ the recurrence relation is satisfied with accuracy of $O(E / n)$ by the ansatz (2.11).

The recurrence relation at large $n$ does not determine the constant $A$. To obtain the value of $A$, one can consider the region of very small $E, E \ll 1$ and make contact with our previous result (2.10) which in the large $n$ limit reads 


$$
A_{n}(E)=n !\left(\frac{\lambda}{8}\right)^{(n-1) / 2}\left(1-\frac{5}{6} E\right)
$$

By comparing (2.11) and (2.15), one finds

$$
A=-\frac{5}{6}
$$

So, we have established the formula (2.2) for the tree amplitude of the production of $n$ nonrelativistic particles. The result has the form expected from the exponentiation hypothesis.

\section{EXPONENTIATION OF LEADING $-n$ CORRECTIONS FROM ALL LOOPS FOR $1 \rightarrow n$ AT THRESHOLD}

\section{A. General formalism}

In this section we consider the process $1 \rightarrow n\left(n\right.$ odd) at threshold in the unbroken $\varphi^{4}$ theory. The amplitude of this process has been calculated in refs. [0, 19,20] at the tree level and in refs. 22 24] in one loop. The result is

$$
A_{n}^{\text {tree }}+A_{n}^{1 \text {-loop }}=A_{n}^{\text {tree }}\left(1+B \lambda n^{2}\right)
$$

where $B$ is a complex number that depends on the number of space-time dimensions. In $(3+1)$ dimensions the value of $B$ is given by eq.(1.14). We will consider higher loop corrections to the leading order in $n$. In the $k$-th loop they are of order $\left(\lambda n^{2}\right)^{k}$ [24]. We will show that these corrections sum up into the exponent

$$
A_{n}=A_{n}^{\text {tree }} \exp \left(B \lambda n^{2}\right)
$$

This result strongly supports the conjecture stated in Introduction.

The technique that we use in this section is based on the formalism of ref. [19] that reduces the problem of the evaluation of the amplitude $1 \rightarrow n$ at the threshold to the calculation of one-leg Feynman graphs in a certain spatially homogeneous classical background field. We perform direct evaluation of Feynman graphs, and show that to the leading order in $n$, one 
can reduce the calculation of loop graphs to a simpler calculation of tree graphs in some effective theory.

Let us outline briefly the technique developed in ref. [19]. For convenience let us again set the mass of the particle $m=1$. Consider the transition from an initial virtual particle with four-momentum $P_{\mu}=(n, 0)$ into $n$ final particles, each with four-momentum $(1,0)$. The reduction formula for the amplitude can be written in the following form,

$$
A_{n}=\lim _{\omega^{2} \rightarrow 1} \lim _{J_{0} \rightarrow 0}\left(\omega^{2}-1\right)^{n} \frac{\partial^{n}}{\partial J^{n}} \int d^{4} x \mathrm{e}^{-i n t}\langle 0|\varphi(x)| 0\rangle_{J=J_{0} \exp (i \omega t)}
$$

where the matrix element is calculated in the presence of the source $J=J_{0} \exp (i \omega t)$.

Let us write the classical field equation with the source,

$$
\partial_{\mu}^{2} \varphi-\varphi-\lambda \varphi^{3}+J_{0} \mathrm{e}^{i \omega t}=0
$$

One of the solutions to this equation has the following expansion,

$$
\varphi_{0}(t)=z(t)+\ldots
$$

where

$$
z(t)=z_{0} \mathrm{e}^{i t} \equiv \frac{J_{0}}{\omega^{2}-1} \mathrm{e}^{i t}
$$

and dots stand for terms proportional to $e^{3 i t}, e^{5 i t}$, etc. The crucial point is that one can take limits $\omega^{2} \rightarrow 1$ and $J_{0} \rightarrow 0$ simultaneously, so that the ratio $z_{0}$ remains finite. In this case, the field equation (3.2) becomes sourceless and its solution having the form (3.3) can be found exactly,

$$
\varphi_{0}(t)=\frac{z(t)}{1-\frac{\lambda}{8} z^{2}(t)}
$$

Taking into acount the following relation which comes from eq.(3.4),

$$
\mathrm{e}^{-i t}\left(\omega^{2}-1\right) \frac{\partial}{\partial J_{0}}=\frac{\partial}{\partial z}
$$

one can rewrite the amplitude in the following form 


$$
A_{n}=\frac{\partial^{n}}{\partial z^{n}}\langle 0|\varphi| 0\rangle
$$

where the matrix element is calculated in the classical background (3.5).

Thus, the problem of evaluating the $1 \rightarrow n$ amplitude at the threshold reduces to the calculation of the matrix element of the field operator in a classical background. This matrix element can be calculated in terms of conventional Feynman graphs. This is the technique invented in ref. $19 \|$.

At the tree level, one replaces the matrix element in eq.(3.6) by the background field (3.5) and finds

$$
A_{n}^{\text {tree }}=n !\left(\frac{\lambda}{8}\right)^{\frac{n-1}{2}} .
$$

To calculate loop corrections, it is convenient to evaluate the matrix element in euclidean space and after that perform the Wick rotation. By introducing the euclidean time variable,

$$
\tau=i t+\frac{1}{2} \ln \frac{\lambda}{8}+\ln z_{0}+i \frac{\pi}{2}
$$

the background configuration in the euclidean space is written as follows,

$$
\varphi_{0}(\tau)=-i \sqrt{\frac{\lambda}{2}} \frac{1}{\cosh \tau} .
$$

Extracting the quantum part $\tilde{\varphi}$ from the field operator $\varphi$,

$$
\varphi=\varphi_{0}+\tilde{\varphi}
$$

one has $\langle 0|\varphi| 0\rangle=\varphi_{0}+\langle 0|\tilde{\varphi}| 0\rangle$. To calculate the matrix element of $\tilde{\varphi}$ one first derives the Feynman rules. For this purpose we write the euclidean lagrangian for $\tilde{\varphi}$,

$$
L=\frac{1}{2}\left(\partial_{\mu} \tilde{\varphi}\right)^{2}+\frac{1}{2}\left(1+3 \lambda \varphi_{0}^{2}\right) \tilde{\varphi}^{2}+\lambda \varphi_{0} \tilde{\varphi}^{3}+\frac{\lambda}{4} \tilde{\varphi}^{4}
$$

from which one obtains the Feynman rules shown in fig. 3 .

Let us denote the contribution from the $k$-th loop by $\varphi_{k}(\tau)$. Since the background field $\varphi_{0} \sim \cosh ^{-1}(\tau)$ is singular at $\tau=i \pi / 2$, one expects that $\varphi_{k}$ is also singular at this value 
of $\tau$. Assuming that the leading singularity is a pole of order $n_{k}$ (in fact, we will see that $\left.n_{k}=2 k+1\right)$, one can expand $\varphi_{k}$ around the singularity in powers of $\varphi_{0}$,

$$
\varphi_{k}=c_{0} \varphi_{0}^{n_{k}}+c_{1} \varphi_{0}^{n_{k}-1}+c_{2} \varphi_{0}^{n_{k}-2}+\ldots
$$

Substituting eq.(3.5) into eq.(3.7) and differentiating $n$ times with respect to $z$, one finds that the leading $n$ behavior of the amplitude is determined by the leading singular term in the right hand side of eq.(3.7). Namely, at large $n$ the contribution from the $k$-th loop to the amplitude will be

$$
\frac{c_{0}}{\left(n_{k}-1\right) !}\left(\frac{2}{\lambda}\right)^{\left(n_{k}-1\right) / 2} n^{n_{k}-1}+O\left(n^{n_{k}-2}\right) .
$$

So, if we wish to calculate, in each order of the perturbation theory, the leading- $n$ contribution only, we do not need to know exactly the function $\varphi_{k}$ but only its leading singularity at $\tau=i \pi / 2$.

At the one-loop level, the only graph that makes contribution to $\langle 0|\tilde{\varphi}| 0\rangle$ is shown in fig. Ala. Analytically, one has

$$
\varphi_{1}(x)=(-3 \lambda) \int d y D\left(x, x^{\prime}\right) \varphi_{0}\left(x^{\prime}\right) D\left(x^{\prime}, x^{\prime}\right)
$$

where $D(x, y)$ is the propagator in the background $\varphi_{0}$. Let us consider it more closely. $D\left(x, x^{\prime}\right)$ satisfies the following differential equation,

$$
\hat{O} D\left(x, x^{\prime}\right) \equiv\left(-\partial_{x}^{2}+1+3 \lambda \varphi_{0}^{2}\right) D\left(x, x^{\prime}\right)=\delta^{4}\left(x-x^{\prime}\right)
$$

The analytic formula for the propagator is known [22,24]. It is convenient to write the propagator in the mixed coordinate-momentum representation (momentum in space, coordinate in time),

$$
D\left(x, x^{\prime}\right)=\int \frac{d^{d} \mathbf{p}}{(2 \pi)^{d}} \mathrm{e}^{i \mathbf{p}\left(\mathbf{x}-\mathbf{x}^{\prime}\right)} D\left(\tau, \tau^{\prime} ; \mathbf{p}\right)
$$

where $d$ is dimension of space and $\tau$ and $\tau^{\prime}$ are time components of $x$ and $x^{\prime}$, respectively. One writes 


$$
D\left(\tau, \tau^{\prime} ; \mathbf{p}\right)=\frac{1}{W_{\mathbf{p}}}\left(f_{1}^{\omega}(\tau) f_{2}^{\omega}\left(\tau^{\prime}\right) \theta\left(\tau^{\prime}-\tau\right)+f_{2}^{\omega}(\tau) f_{1}^{\omega}\left(\tau^{\prime}\right) \theta\left(\tau-\tau^{\prime}\right)\right)
$$

where $f_{1}^{\omega}(\tau)$ and $f_{2}^{\omega}(\tau)$ are two linear independent solutions to the homogeneous differential equation

$$
\left(-\partial_{\tau}^{2}+\omega^{2}-\frac{6}{\cosh ^{2} \tau}\right) f(\tau)=0
$$

with $\omega=\sqrt{\mathbf{p}^{2}+1} . f_{1}^{\omega}(\tau)$ and $f_{2}^{\omega}(\tau)$ tend to zero as $\tau \rightarrow-\infty$ and $\tau \rightarrow \infty$, respectively,

$$
\begin{gathered}
f_{1}^{\omega}(\tau)=\mathrm{e}^{\omega \tau}\left(\omega^{2}-3 \omega \tanh \tau+2-\frac{3}{\cosh ^{2} \tau}\right) \\
f_{2}^{\omega}(\tau)=f_{1}^{-\omega}(\tau)
\end{gathered}
$$

In eq.(3.9) $W_{\mathbf{p}}=f_{1}^{\prime} f_{2}-f_{2}^{\prime} f_{1}$ is their Wronskian,

$$
W_{\mathbf{p}}=2 \omega\left(\omega^{2}-1\right)\left(\omega^{2}-4\right)
$$

In what follows it will be more convenient to work with new functions $f$ and $g$ which are linear combinations of the old ones,

$$
\begin{gathered}
f_{1}(\tau)=\mathrm{e}^{i \pi \omega / 2}(f(\tau)+g(\tau)) \\
f_{2}(\tau)=\mathrm{e}^{-i \pi \omega / 2}(f(\tau)-g(\tau))
\end{gathered}
$$

The functions $f$ and $g$ have different behavior around $\tau=i \pi / 2$ : while $f$ is singular, $f(\tau)=$ $-3 \cosh ^{-2} \tau$, the function $g$ is regular at this point, $g(\tau) \sim \cosh ^{3} \tau$. In terms of $f$ and $g$, the propagator can be represented in the following form,

$$
D\left(\tau, \tau^{\prime} ; \mathbf{p}\right)=D_{0}\left(\tau, \tau^{\prime} ; \mathbf{p}\right)+D_{1}\left(\tau, \tau^{\prime} ; \mathbf{p}\right)
$$

where

$$
D_{0}\left(\tau, \tau^{\prime} ; \mathbf{p}\right)=\frac{1}{W_{\mathbf{p}}} f(\tau) f\left(\tau^{\prime}\right)
$$

and 


$$
D_{1}\left(\tau, \tau^{\prime} ; \mathbf{p}\right)=\frac{1}{W_{\mathbf{p}}}\left[\epsilon\left(\tau-\tau^{\prime}\right)\left(f(\tau) g\left(\tau^{\prime}\right)-f\left(\tau^{\prime}\right) g(\tau)\right)-g(\tau) g\left(\tau^{\prime}\right)\right]
$$

$D_{0}$ contains the strongest singularity of $D$, while $D_{1}$ is less singular than $D_{0}$. Note that $D_{0}\left(\tau, \tau^{\prime}, \mathbf{p}\right)$ factorizes, this fact will be extensively explored in what follows. We will need the following formula for $D_{0}$ which is correct in the sense of the leading singularity,

$$
D_{0}\left(\tau, \tau^{\prime} ; \mathbf{p}\right) \cong \frac{9}{4 W_{\mathbf{p}}} \varphi_{0}^{2}(\tau) \varphi_{0}^{2}\left(\tau^{\prime}\right)
$$

Let us consider the propagator at coinciding points that enters eq.(3.8). The leading singularity in $D(x, x)$ is

$$
\begin{aligned}
& D(x) \equiv D(x, x) \cong \int \frac{d^{d} \mathbf{p}}{(2 \pi)^{d}} \frac{1}{2 \omega\left(\omega^{2}-1\right)\left(\omega^{2}-4\right)} f^{2}(\tau) \\
& =\int \frac{d^{d} \mathbf{p}}{(2 \pi)^{d}} \frac{9}{2 \omega\left(\omega^{2}-1\right)\left(\omega^{2}-4\right)} \frac{1}{\cosh ^{4}(\tau)}=\lambda^{2} B \varphi_{0}^{4}(\tau)
\end{aligned}
$$

where we introduced the notation

$$
B=\int \frac{d^{d} \mathbf{p}}{(2 \pi)^{d}} \frac{9}{8 \omega\left(\omega^{2}-1\right)\left(\omega^{2}-4\right)}
$$

Now we are able to calculate $\varphi_{1}$. Acting by the operator $\hat{O}$ on both sides of eq.(3.8) and taking into accound the relation $\hat{O} D\left(x, x^{\prime}\right)=\delta\left(x-x^{\prime}\right)$, one finds

$$
\hat{O} \varphi_{1}=-3 \lambda^{3} B \varphi_{0}^{5}
$$

To solve this equation let us notice that

$$
\begin{aligned}
& \hat{O} \varphi_{0}^{n}=\left(-\partial_{\mu}^{2}+1-\frac{6}{\cosh ^{2} \tau}\right) i^{n}\left(\frac{2}{\lambda}\right)^{n / 2} \frac{1}{\cosh ^{n} \tau} \\
& =i^{n}\left(\frac{2}{\lambda}\right)^{n / 2} \frac{(n-2)(n+3)}{\cosh ^{n+2} \tau}+O\left(1 / \cosh ^{n} \tau\right) \\
& =-\left(\frac{\lambda}{2}\right)(n-2)(n+3) \varphi_{0}^{n+2}+\text { subleading terms }
\end{aligned}
$$

where only the term with the strongest singularity is written explicitly. Reversing this equation we obtain 


$$
\begin{gathered}
\left(\hat{O}^{-1} \varphi_{0}^{n+2}\right)(x)=\int d^{d} y D(x, y) \varphi_{0}^{n+2}(y) \\
\cong-\frac{2}{(n-2)(n+3)} \frac{1}{\lambda} \varphi_{0}^{n}
\end{gathered}
$$

Applying this formula to eq.(3.12) one finds, in the sense of leading singularity,

$$
\varphi_{1}=\lambda^{2} B \varphi_{0}^{3}
$$

So, the amplitude up to one-loop is

$$
A_{n}=A_{n}^{\text {tree }}\left(1+B \lambda n^{2}+O(\lambda n)\right)
$$

which coincides with the result obtained in refs. 22,24], eq.(1.13).

So, we have seen that the one loop correction can be evaluated without cumbersome calculations. Let us turn to the two-loop level.

\section{B. Two-loop level}

In the previous subsection we have calculated the tadpole of fig. tha. In our calculation we have replaced the propagator at coinciding points, $D\left(x^{\prime}, x^{\prime}\right)$, by its leading singular part which is proportional to $\varphi_{0}^{4}$. For further convenience, we represent the amplitude after this replacement by the graph shown in fig. $4 \mathrm{~b}$, which can be obtained from fig. 19a by cutting the inner line. Each line that ends on a bullet corresponds to a factor of $B^{1 / 2} \lambda \varphi_{0}^{2}$.

There are six different graphs that contribute to the two-loop amplitude (see fig.5 $5 \mathrm{a}-\mathrm{f}$ ). The first two graphs are easy to calculate by using the same technique as in the one-loop case. One writes for the first graph (hereafter equalities are to be understood in the sense of leading singularities)

$$
\varphi_{2 a}=-3 \lambda \hat{O}^{-1} \varphi_{0} \varphi_{1}^{2}
$$

Substituing $\varphi_{1}$ from eq.(3.14) into this equation and applying the formula (3.13), one obtains

$$
\varphi_{2 a}=\frac{1}{8} \lambda^{4} B^{2} \varphi_{0}^{5}
$$


Analogously, for the second graph, fig.Ðb, one has

$$
\varphi_{2 b}=-6 \lambda \hat{O}^{-1} \varphi_{1} D=\frac{1}{4} \lambda^{4} B^{2} \varphi_{0}^{5}
$$

Graphically, one can represent the first two graphs in the new form as shown in the first two equations of fig. 5 .

Let us consider the third graph. One writes

$$
\left(\hat{O} \varphi_{2 c}\right)(x)=18 \lambda^{2} \varphi_{0}(x) \int d y D^{2}(x, y) \varphi_{0}(y) \varphi_{1}(y)
$$

At first sight, to find the leading singularity in $\varphi_{2 c}$, it appears to be natural to suppose that $D(x, y)$ can be replaced by $D_{0}(x, y)$. In this way one would have

$$
\left(\hat{O} \varphi_{2 c}\right)(x) \sim \varphi_{0}(\tau) f^{2}(\tau) \int \frac{d^{d} \mathbf{p}}{(2 \pi)^{3}} \frac{f^{2}\left(\tau^{\prime}\right) \varphi_{0}\left(\tau^{\prime}\right) \varphi_{1}\left(\tau^{\prime}\right)}{W_{\mathbf{p}}^{2}}
$$

The integral is some constant, so one would obtain

$$
\left(\hat{O} \varphi_{2 c}\right)(x) \sim \varphi_{0}^{5}(\tau)
$$

where we have made use of the relation $f(\tau) \sim \varphi_{0}^{2}(\tau)$. So, one would conclude that $\varphi_{2 c} \sim$ $\varphi_{0}^{3}$ which is less singular than the result for the graphs $5 \mathrm{a}$ and $5 \mathrm{~b}, \varphi_{0}^{5}$. However, this argumentation is wrong. The reason is that $D_{0}\left(\tau, \tau^{\prime}\right)$ is smooth at $\tau=\tau^{\prime}$, so that the contribution proportional to $D_{0}^{2}$ into the right hand side of eq.(3.15) is not too singular. More singular contribution comes from the term $D_{0} D_{1}$,

$$
\left(\hat{O} \varphi_{2 c}\right)(x)=36 \lambda^{2} \varphi_{0}(x) \int d y D_{0}(x, y) D_{1}(x, y) \varphi_{0}(y) \varphi_{1}(y)
$$

Since the contribution with $D_{0}^{2}$ in the integrand is more regular, one can replace $D_{1}$ by $D$ and not spoil the leading singular behavior,

$$
\left(\hat{O} \varphi_{2 c}\right)(x)=36 \lambda^{2} \varphi_{0}(x) \int d y D_{0}(x, y) D(x, y) \varphi_{0}(y) \varphi_{1}(y)
$$

Switching to the mixed representation and using eq.(3.10), one obtains

$$
\left(\hat{O} \varphi_{2 c}\right)(x)=36 \lambda^{2} \varphi_{0}^{3}(x) \int \frac{d^{d} \mathbf{p}}{(2 \pi)^{3}} d \tau \frac{9}{W_{\mathbf{p}}} D\left(\tau, \tau^{\prime} ; \mathbf{p}\right) \varphi_{0}^{3}\left(\tau^{\prime}\right) \varphi_{1}\left(\tau^{\prime}\right)
$$


Let us first take the integral over $d \tau^{\prime}$. Since one is interested only in the leading singularity, one can replace $D\left(\tau, \tau^{\prime} ; \mathbf{p}\right)=\left(-\partial_{\tau}^{2}+\omega^{2}-6 \cosh ^{-2} \tau\right)^{-1}\left(\tau, \tau^{\prime}\right)$ by $\hat{O}^{-1}=\left(-\partial^{2}+1-\right.$ $\left.6 \cosh ^{-2} \tau\right)^{-1}$, since 1 and $\omega$ are both more regular than $\partial^{2}$ and $\cosh ^{-2} \tau$. The integral over $d^{3} \mathbf{p}$ gives an additional factor $(-B)$, so one has

$$
\varphi_{2 c}=-36 \lambda^{4} B \hat{O}^{-1} \varphi_{0}^{3} \hat{O}^{-1} \varphi_{0}^{3} \varphi_{1}
$$

Making use of the formula for $\varphi_{1}$, eq.(3.14), and the rule (3.13), one obtaines finally

$$
\varphi_{2 c}=\frac{3}{7} \lambda^{4} B^{2} \varphi_{0}^{5}
$$

Let us now turn to the graphic interpretation of eq.(3.18). The corresponding graph is shown in fig.5 $5 \mathrm{c}^{\prime}$. The solid line represents the operator $\hat{O}^{-1}$, which originates from the full propagator in fig. $5 \mathrm{f}^{\prime}$, while each line ending with a bullet comes from cutting a propagator and corresponds to a factor of $\lambda B^{1 / 2} \varphi_{0}^{2}$. The additional factor 2 comes from the two possible internal lines we can cut. It is instructive to learn which graph corresponds to eq.(3.16). This graph is shown in fig.6: it can be obtained from the initial graph by cutting both internal propagators. Fig.6 is a disconnected graph, and it is clear that it is proportional

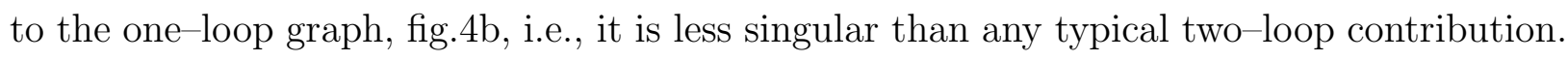
Hence this graph can be neglected.

Now it is clear how to evaluate the remaining graphs in fig.5. One must cut maximum number of internal lines in such a way that the obtained graph is tree, but still remains connected. If a graph can be cut in different ways, all the obtained graphs make contributions. For instance, for the graph of fig.5f, there are five different ways to cut: one can cut the line 1 and 3,1 and 4,2 and 3,2 and 4, or 3 and 4 .

To make our presentation more explicit, we write down here the result for all two-loop graphs (fig.5)

$$
\begin{aligned}
& \varphi_{2 a}=\frac{1}{2}(-6 \lambda) \hat{O}^{-1} \varphi_{0} \varphi_{1}^{2}, \\
& \varphi_{2 b}=\frac{1}{2}\left(6 \lambda^{3} B\right) \hat{O}^{-1} \varphi_{0}^{4} \varphi_{1},
\end{aligned}
$$




$$
\begin{gathered}
\varphi_{2 c}=-36 \lambda^{4} B \hat{O}^{-1} \varphi_{0}^{3} \hat{O}^{-1} \varphi_{0}^{3} \varphi_{1}, \\
\varphi_{2 d}=\frac{1}{2}\left(36 \lambda^{6} B^{2}\right) \hat{O}^{-1} \varphi_{0}^{3} \hat{O}^{-1} \varphi_{0}^{6}, \\
\varphi_{2 e}=\left(6 \lambda^{3} B\right) \hat{O}^{-1} \varphi_{0}^{4} \varphi_{1}, \\
\varphi_{2 f}=(-6 \lambda) \hat{O}^{-1} \varphi_{0} \varphi_{1}^{2}-2\left(36 \lambda^{4} B\right) \hat{O}^{-1} \varphi_{0}^{3} \hat{O}^{-1} \varphi_{0}^{3} \varphi_{1} .
\end{gathered}
$$

Summing up the contributions from all graphs, one obtains the total two-loop correction to the generating function,

$$
\begin{gathered}
\varphi_{2}=\frac{3}{2}(-6 \lambda) \hat{O}^{-1} \varphi_{0} \varphi_{1}^{2}+ \\
\frac{3}{2}\left(6 \lambda^{3} B\right) \hat{O}^{-1} \varphi_{0}^{4} \varphi_{1}-3\left(36 \lambda^{4} B\right) \hat{O}^{-1} \varphi_{0}^{3} \hat{O}^{-1} \varphi_{0}^{3} \varphi_{1}+ \\
3 \frac{1}{6}\left(36 \lambda^{6} B^{2}\right) \hat{O}^{-1} \varphi_{0}^{3} \hat{O}^{-1} \varphi_{0}^{6} .
\end{gathered}
$$

Making use of the explicit formula for $\varphi_{1}$ and the rule (3.13), one finds

$$
\varphi_{2}=3 \lambda^{4} B \varphi_{0}^{5}
$$

(We have checked this result by direct - and tedious - calculation of the Feynman graphs.) Thus, the leading $-n$ threshold amplitude up to the two loop level is

$$
A_{n}^{\text {tree }}+A_{n}^{1 \text {-loop }}+A_{n}^{2 \text {-loop }}=A_{n}^{\text {tree }}\left(1+B \lambda n^{2}+\frac{B^{2}}{2} \lambda^{2} n^{4}\right),
$$

We see that, up to the two-loop level, the leading- $n$ correction coincides with the exponent $\exp \left(B \lambda n^{2}\right)$. In the next subsection we show the exponentiation in all orders of the perturbation theory.

\section{Exponentiation}

In the previous subsection we have designed a technique that reduces the calculation of the leading $-n$ behavior of any Feynman graph to a simple arithmetic operations. The 
prescription is to cut a maximum number of internal lines to obtain a set of connected tree graphs with external lines. Each external line that comes from an internal propagator is then replaced by a factor of $\lambda B^{1 / 2} \varphi_{0}^{2}$, and each propagator that has not been cut is associated with the operator $\hat{O}^{-1}$. This procedure allows us to calculate the leading singularity in an arbitrary Feynman graph.

Consider the $k$-loop level. Since the new graph obtained by cutting is tree, it is clear that one must cut $k$ propagators. So, the constant $B$ appears $k$ times. By a simple counting argument one can show that every $k$-loop graph is proportional to $\lambda^{2 k} B^{k} \varphi_{0}^{2 k+1}$. Thus, the generating function to all orders of perturbation theory can be written in the following form

$$
\langle 0|\varphi| 0\rangle=\varphi_{0} \sum_{k=0}^{\infty} d_{k}\left(\lambda^{2} B \varphi_{0}^{2}\right)^{k}
$$

where $d_{k}$ are some constant.

According to eq.(3.6), this formula implies the following leading $-n$ expansion for the amplitude $A_{n}$,

$$
A_{n}=A_{n}^{\text {tree }}\left(1+\sum_{k=1}^{\infty} \frac{2^{k} d_{k}}{(2 k) !} \lambda^{k} B^{k} n^{2 k}\right)
$$

To see that the whole sum (3.20) is in fact the exponent, we have to show that $d_{k}=$ $(2 k) ! /\left(2^{k} k !\right)$. There are at least two ways to see that this is indeed the case. The first one is indirect and is based on the fact that the coefficients $d_{k}$ do not depend on the dimensionality of space-time (in our techniques, the dimensionality of space-time enters only through the constant $B$ that is determined by eq.(3.11)). So, to calculate the constants $d_{k}$ one can work in any space-time dimension. One notices that in $(2+1)$ dimensions the integral that defines the constant $B$, eq.(3.11), diverges logarithmically in the infrared region (see section [V). One can regularize the integral by introducing, for example, finite spatial volume $V$. In this case the series (3.19) is in fact a sum of leading logarithms. Comparing this series with the leading-log result of section $\mathbb{\mathbb { V }}$, one finds that the right hand side of eq.(3.20) in fact sums up to an exponent.

The second way is a direct analysis of the perturbative series. First, we note that the graphs obtained after the cutting procedure have the same form as the tree graphs in an 
effective theory where the condensate $\varphi_{0}$ is shifted by $\lambda B^{1 / 2} \varphi_{0}^{2}$, with the only difference in the symmetry factor: each graph with $2 k$ external lines ending at bullets must be multiplied by a factor of $(2 k) ! /\left(2^{k} k !\right)$. So, the prescription is as follows. One searches for a solution to the classical field equation (which is equivalent to the summation of tree graphs),

$$
\partial_{\tau}^{2} \varphi=\lambda \varphi^{3}
$$

(the mass can be neglected) which has the form

$$
\varphi_{\mathrm{cl}}=\varphi_{0}+\lambda B^{1 / 2} \varphi_{0}^{2}+\ldots
$$

The meaning of ... in this formula is explained in the explicit expansion of $\varphi_{c l}$,

$$
\varphi_{\mathrm{cl}}=\varphi_{0} \sum_{k=0}^{\infty} \alpha_{k}\left(\lambda B^{1 / 2} \varphi_{0}\right)^{k}
$$

where $\alpha_{k}$ are some numerical coefficients, $\alpha_{0}=\alpha_{1}=1$. For $k>1$ the coefficient $\alpha_{k}$ can be calculated perturbatively and is given by graphs with $k$ external lines ending at bullets. Since $d_{k}$ are also given by graphs with $2 k$ lines ending at bullets, and because of the difference in symmetry factors in the two theories, this analogy implies the following relation between the coefficients $d_{k}$ in eq. (3.19) and $\alpha_{k}$ in eq.(3.22),

$$
d_{k}=\frac{(2 k) !}{2^{k} k !} \alpha_{2 k}
$$

We are interested in the region around the singularity $\tau=i \pi / 2$, so in eq.(3.22) one can replace $\varphi_{0}$ by its singular part

$$
\varphi_{0} \rightarrow \sqrt{\frac{2}{\lambda}} \frac{1}{\tau-i \pi / 2}
$$

and obtain

$$
\varphi_{\mathrm{cl}}=\sqrt{\frac{2}{\lambda}} \frac{1}{\tau-i \pi / 2}+\frac{2 B^{1 / 2}}{(\tau-i \pi / 2)^{2}}+\sum_{k=2}^{\infty} \alpha_{k}\left(\lambda B^{1 / 2}\right)^{k}\left(\sqrt{\frac{2}{\lambda}} \frac{1}{\tau-i \pi / 2}\right)^{k+1}
$$

where we have written down explicitly the first two terms of the sum. On the other hand, one can verify that 


$$
\varphi(\tau)=\frac{2}{\lambda} \frac{1}{\tau-i \pi / 2-\sqrt{2 \lambda B}}
$$

is a solution to eq.(3.21) and it has the same form as indicated in (3.24). Comparing eqs.(3.25) and (3.24), one obtains $\alpha_{k}=1$. From (3.23) we see that $d_{k}=(2 k) ! /\left(2^{k} k !\right)$, so the leading $-n$ amplitude, eq.(3.20), is indeed exponential.

So, we have shown that leading $-n$ corrections sum up into the exponent. We note finally that the whole calculation of this section can be applied to the $\varphi^{4}$ model with reflection symmetry breaking without any major modification. The leading $-n$ loop corrections also sum up into the exponent, but in the broken case the constant $B$ is real and positive. So, instead of being reduced, the amplitude is enhanced by a factor of $\exp \left(B \lambda n^{2}\right)$. Whether this enhancement persists when one includes more terms in the expansion of the function $F(\lambda n)$ is still an open question.

\section{RENORMALIZATION GROUP FOR MULTIPARTICLE PRODUCTION IN (2+1) DIMENSIONS NEAR THE THRESHOLD}

\section{A. Unbroken $\varphi^{4}$ theory}

In this section we consider the multiparticle production in $(2+1)$ dimensions, where the infrared divergencies exist in a certain kind of loop graphs, which break the conventional pertubative expansion in a region close enough to the threshold. The graphs of this kind contain loops related to the rescattering among final soft particles. It is a peculiar feature of $(2+1)$ dimensions that right at the threshold, rescattering graphs diverge logarithmically, so even at small number of final particles $n$, the calculation of the amplitudes around the threshold requires a nontrivial summation of an infinite set of graphs.

We describe a technique to perform this summation which is a modification of the conventional renormalization group (RG). This technique allows us to sum up leading logarithms from all orders of the perturbation theory. By this technique we obtain the amplitude 
$1 \rightarrow n$ in the $\varphi^{4}$ models with both broken and unbroken reflection symmetry, and in the $O(N)$ theory.

Let us consider the $\varphi^{4}$ theory in $(2+1)$ dimensions,

$$
L=\frac{1}{2}\left(\partial_{\mu} \varphi\right)^{2}-\frac{1}{2} \varphi^{2}-\frac{\lambda}{4} \varphi^{4}
$$

where, as before, we set the mass of the particle equal to one. This is the theory of interacting relativistic bosons.

To describe bosons in the low-energy limit, one writes the following effective lagrangian in terms of a non-relativistic bosonic field $\Psi$,

$$
L_{\mathrm{eff}}=\Psi^{\dagger} i \partial_{0} \Psi-\frac{1}{2}\left(\partial_{i} \Psi^{\dagger}\right)\left(\partial_{i} \Psi\right)-g \Psi^{\dagger} \Psi^{\dagger} \Psi \Psi
$$

where $g$ is some, yet to be determined, effective coupling.

Non-relativistic bosons, interacting via a delta-like potential (as in eq.(4.1)), have been known for long time as an example of a non-relativistic system with dimensional transmutation [27]. In fact, the counting of dimensions in $(2+1) \mathrm{d}$ (in non-relativistic kinematics the relation between the dimensions of energy and momentum is $[E]=[p]^{2}$ ) implies that $\Psi^{\dagger} \Psi^{\dagger} \Psi \Psi$ is a marginal operator and the coupling constant $g$ is dimensionless. Apparently, there is no scale parameter in the theory described by the lagrangian (4.1). However, this scale exists and is merely the boson mass (which is equal to one in our notation), which plays the role of the "ultraviolet" cutoff in the effective theory.

To make contact between the effective lagrangian and the initial Lorentz-invariant one, one compares formulas for the amplitude of elastic scattering of two bosons computed in both theories. This results in the following relation between $g$ and $\lambda$,

$$
g=3 \lambda / 8
$$

Considering $g$ as the bare coupling, or the coupling at the scale of the ultraviolet cutoff, one can study the evolution of $g$ as a function of the momentum scale. For this purpose we introduce the running coupling constant $g(t)$, which has the physical meaning of the 
strength of the interaction between bosons at the momentum scale $p=\mathrm{e}^{-t},(t=\ln (1 / p))$, or, equivalently, the kinetic energy

$$
\epsilon=\mathrm{e}^{-2 t}
$$

There is only one Feymnan diagram (shown in fig.7) that makes contribution to the corresponding beta function. Simple calculation yields the following RG equation,

$$
\frac{d g(t)}{d t}=-\frac{g^{2}(t)}{\pi}
$$

which has the solution

$$
g(t)=\frac{g}{1+\frac{g}{\pi} t}=\frac{3 \lambda}{8}\left(1+\frac{3 \lambda}{8 \pi} t\right)^{-1}
$$

where we have made use of the initial condition for $g(t), g(0)=g$, and $g$ is defined by eq.(4.2). From eq.(4.3) one sees that the strength of the interaction between bosons decreases as the momenta of particles tend to zero. Later, we will demonstrate that this property holds for a more general case of $O(N)$ model, while in the theory with symmetry breaking the behavior of $g(t)$ is just the opposite.

The coupling constant changes considerably from its initial value only when the momentum scale is exponentially small in $\lambda$, i.e., $t=\ln (1 / p) \sim 1 / \lambda$. So, the renormalization group is suitable for considering the regime $\lambda \rightarrow 0, \lambda t \sim 1$. All further considerations in this section will be done for this particular regime.

In fact, the flow of the effective coupling, eq.(4.3), can be obtained by a simpler method of direct summation of bubble graphs: in non-relativistic theories only these graphs contribute to the elastic scattering $2 \rightarrow 2$. However, in the calculation of the amplitudes of multiparticle production near the threshold more complicated diagrams are involved and the problem cannot be reduced to the summation of bubble graphs. For example, for the $1 \rightarrow 3$ process the one- and two-loop diagrams that make contribution in our leading-log regime are presented in fig.8. To deal with these processes the RG technique is essential. In the diagrammatic language, the renormalization group corresponds to the summation of leading logarithms, i.e., terms proportional to $(\lambda t)^{k}$ in the whole perturbation series. 
Let us try to describe the production of $n$ final particles in terms of the non-relativistic bosonic creation operator $\Psi^{\dagger}$. The only relevant candidate is $A_{n} \Psi^{\dagger n}$. The following relation should take place,

$$
\langle n|\varphi| 0\rangle=\left\langle n\left|A_{n} \Psi^{\dagger n}\right| 0\right\rangle_{\mathrm{eff}},
$$

where the matrix element in the left hand side is written in the initial $\varphi^{4}$ model and the right hand side is understood as a matrix element in the effective non-relativistic theory. From this equation one finds that $A_{n}$ is equal to the $1 \rightarrow n$ amplitude when the spatial momenta of final particles are small, but not exponentially small (so the logarithms do not appear in loops). Let us for simplicity consider small enough $n$ where $A_{n}$ coincides with the tree $1 \rightarrow n$ amplitude [7, 19.20],

$$
A_{n}=A_{n}^{\text {tree }}=n !\left(\frac{\lambda}{8}\right)^{(n-1) / 2} .
$$

When the characteristic scale of momenta of final particles is exponential, the right hand side of eq.(4.4) is substantially renormalized by loops. One can treat this renormalization by introducing the running vertex $A_{n}(t)$ and solving the corresponding RG equation,

$$
\frac{d A_{n}(t)}{d t}=-\frac{n(n-1)}{2} \frac{1}{\pi} g(t) A_{n}(t) .
$$

Graphically, this equation is represented in fig.9. Substituting the function $g(t)$ given by eq.(4.3), to eq.(4.5), we obtain the function $A_{n}(t)$,

$$
A_{n}(t)=A_{n}\left(1+\frac{g(0)}{\pi} t\right)^{-\frac{n(n-1)}{2}}=A_{n}^{\text {tree }}\left(1+\frac{3 \lambda}{8 \pi} t\right)^{-\frac{n(n-1)}{2}} .
$$

So, we have found that the $\mathrm{RG}$ technique allows us to calculate the $1 \rightarrow n$ amplitude $A_{n}(t)$ in a region close to the threshold where $\lambda t \sim 1$. Note that exactly at the threshold, i.e., at $t=+\infty$, the amplitude vanishes.

\section{B. Broken $\varphi^{4}$ theory}

The above analysis is equally applicable to the case of broken symmetry, 


$$
L=\frac{1}{2}\left(\partial_{\mu} \varphi\right)^{2}-\frac{\lambda}{4}\left(\varphi^{2}-v^{2}\right)^{2},
$$

with the only exception that for evaluating the bare coupling $g$ entering into the lagrangian of the effective theory one should take into account not only the diagram with a four-boson vertex, but also diagrams with two three-boson vertices, i.e. those with the exchange of a virtual particle in $s^{-}, t-$ and $u$-channels. In contrast to the unbroken case, the resulting amplitude is negative, which means the attractive character of the force between bosons at low energies. One has

$$
g=-\frac{3 \lambda}{2}
$$

(we assume $v^{2}=(2 \lambda)^{-1}$, so the mass of the boson is equal to one). Eq.(4.3) implies that the strength of the interaction increases with $t$,

$$
g(t)=\frac{g}{1+\frac{g}{\pi} t}=-\frac{3 \lambda}{2}\left(1-\frac{3 \lambda}{2 \pi} t\right)^{-1} .
$$

Taken at face value, eq.(4.7) predicts infinite coupling constant at exponentially small momentum scale,

$$
p_{0}=\exp (-2 \pi / 3 \lambda) .
$$

This fact is a direct analog of the Landau pole in field theories without asymptotic freedom. In reality, it is a manifestation of the existence of a two-particle bound state in our model (recall that at least one bound state exists in every, arbitrarily weak, two-dimensional attractive potential). One can show that in our case, the energy of the bound state is of order $p_{0}^{2}$.

The $1 \rightarrow n$ amplitude is now

$$
A_{n}(t)=A_{n}^{\text {tree }}\left(1-\frac{3 \lambda}{2 \pi} t\right)^{-\frac{n(n-1)}{2}} .
$$

Let us note in passing, that we may compare our result for the case of large $n, n \gg 1$, to that obtained in ref. [28] for $(2+1)$ dimensions by a different method. In the case when $\lambda t$ is small, $\lambda t \ll 1$, our formula in fact reproduces the result of ref. [28], 


$$
A_{n}=A_{n}^{\text {tree }} \exp \left(\frac{3 \lambda}{4 \pi} n^{2} t\right)
$$

However, if $\lambda t$ is comparable to one, eq.(4.8) does not coincide with that obtained in ref. [28]. We consider this fact as a counter-argument to the claim of ref. 28].

\section{C. $O(N)$ theory}

The application of the technique described above to the multi-component $\varphi^{4}$ model requires a slight modification. The lagrangian of the $O(N)$ model,

$$
L=\frac{1}{2}\left(\partial_{\mu} \varphi_{a}\right)\left(\partial_{\mu} \varphi_{a}\right)-\frac{1}{2} \varphi_{a} \varphi_{a}-\frac{\lambda}{4}\left(\varphi_{a} \varphi_{a}\right)^{2}
$$

( $a=1 \ldots N$ is the internal index) contains one coupling constant $\lambda$. However, if one tries to write down the most general $O(N)$ symmetric effective non-relativistic lagrangian, one sees that there may exist two different effective couplings, $g_{1}$ and $g_{2}$, which correspond to the two possible structures of the potential term,

$$
L_{\mathrm{eff}}=\Psi_{a}^{\dagger} i \partial_{0} \Psi_{a}-\frac{1}{2}\left(\partial_{i} \Psi_{a}^{\dagger}\right)\left(\partial_{i} \Psi_{a}\right)-g_{1} \Psi_{a}^{\dagger} \Psi_{a}^{\dagger} \Psi_{b} \Psi_{b}-2 g_{2} \Psi_{a}^{\dagger} \Psi_{b}^{\dagger} \Psi_{a} \Psi_{b}
$$

$g_{1}$ is the low-energy elastic scattering scattering amplitude, singlet in the $s$-channel, while $g_{2}$ determines amplitudes, singlet in $t$ - and $u$-channels. From the initial lagrangian (4.9) one obtains the bare value of the coupling constants,

$$
g_{1}=g_{2}=\frac{\lambda}{8}
$$

The fact that the bare values of $g_{1}$ and $g_{2}$ are equal to each other is the remnant of the crossing symmetry of our intial lagrangian (4.9). However, the evolution equations for the running coupling constants are different,

$$
\begin{gathered}
\frac{d g_{1}(t)}{d t}=\frac{1}{\pi}\left[N g_{1}^{2}(t)+4 g_{1}(t) g_{2}(t)\right] \\
\frac{d g_{2}(t)}{d t}=\frac{2}{\pi} g_{2}^{2}(t) .
\end{gathered}
$$


In order to simplify the RG equations, let us introduce, instead of $g_{1}(t)$, a linear combination of the two couplings,

$$
g(t)=g_{1}(t)+\frac{2}{N} g_{2}(t)
$$

The RG equation for $g(t)$ is simpler than that of $g_{1}(t)$, eq.4.12),

$$
\frac{d g(t)}{d t}=\frac{N}{\pi} g^{2}(t)
$$

The solution to the RG equations, eqs.(4.13) and (4.14), which satisfies the initial condition (4.11), can then be found,

$$
\begin{gathered}
g_{2}(t)=\frac{\lambda}{8}\left(1+\frac{\lambda}{4 \pi} t\right)^{-1}, \\
g(t)=\left(1+\frac{2}{N}\right) \frac{\lambda}{8}\left(1+(N+2) \frac{\lambda}{8 \pi} t\right) .
\end{gathered}
$$

In analogy to the simple $\varphi^{4}$ case, the production of $n$ soft bosons from an initial particle with isospin $a$ can be described by an effective operator $A_{n} \Psi_{a}^{\dagger}\left(\Psi_{b}^{\dagger} \Psi_{b}^{\dagger}\right)^{(n-1) / 2}(n$ must be odd). After some calculations we obtain the following RG equation for $A_{n}(t)$,

$$
\frac{d A_{n}(t)}{d t}=\frac{1}{\pi}\left[\frac{n-1}{2}(N+n-1) g(t)+(n-1)^{2}\left(1-\frac{1}{N}\right) g_{2}(t)\right] A_{n}(t) .
$$

Having substituted the formulas for $g_{2}(t)$ and $g(t)$ into eq.(4.15) and solved it, one obtains the dependence of the $1 \rightarrow n$ amplitude on the logarithm of the characteristic momentum of final particles,

$$
A_{n}(t)=A_{n}^{\text {tree }}\left(1+(N+2) \frac{\lambda}{8 \pi} t\right)^{-\frac{n-1}{2 N}(N+n-1)}\left(1+\frac{\lambda}{4 \pi} t\right)^{-\frac{(n-1)^{2}}{2 N}(N-1)}
$$

where, as before

$$
t=\ln \left(\frac{1}{p}\right)=\frac{1}{2} \ln \left(\frac{1}{\epsilon}\right)
$$

Recall that the regime which we are describing is $\lambda \rightarrow 0, \lambda t \sim 1$, provided other parameters such as $n$ and $N$ are fixed. 
Eqs.(4.6), (4.8) and (4.16) are our final leading logarithmic expressions for the amplitudes $1 \rightarrow n$. As discussed in Introduction, they have the exponential form at $\lambda t \ll 1$.

To end up this section, let us consider the large $N$ limit and show that the result of ref. [25] can be reobtained in $(2+1)$ dimensions by our technique. Consider the case when $n$ is much smaller than both the number of boson flavors $N$ and the inverse coupling constant. Then eq.(4.16) reduces to a simpler formula,

$$
A_{n}(t)=A_{n}^{\text {tree }}\left[1+\frac{N \lambda}{8 \pi} t\right]^{-(n-1) / 2} .
$$

Recalling that the coupling constant enters into the tree amplitude through the factor $\lambda^{(n-1) / 2}$, one can rewrite eq.(4.17) in the following form,

$$
A_{n}(t) \sim n ! \lambda_{R}^{(n-1) / 2},
$$

where

$$
\lambda_{R}=\frac{\lambda}{1+\frac{N \lambda}{8 \pi} t}
$$

is just the renormalized singlet scattering amplitude. One sees that for a small number of final particles, $n \ll N$, leading order in $1 / N$ result is given just by the tree-level formula where the coupling constant is replaced by the renormalized one. This is precisely the result of ref. [25] in the particular case of $(2+1)$ dimensions.

However, eq.(4.18) is valid only when $n \ll N$. If the number of final particles is comparable to $N$, the effect of loops is obviously not a simple renormalization of the coupling constant. One finds from eq.(4.16) that the correction to the large $-N$ result, eq.(4.17), is proportional to $n^{2} / N$. When the number of final particles becomes comparable to the number of their spieces, the $1 / N$ expansion becomes unreliable. One can expect that the breakdown of the $1 / N$ expansion is not a peculiar feature of $(2+1)$ dimensions but holds also in (3+1)- and higher-dimensional theories.

So, we see that the renormalization group is a poweful mean for investigating the multiparticle amplitudes in $(2+1)$ dimensional scalar field theory at and around the threshold. 
The exact formula for the amplitude, if ever be found, must incorporate the information obtained here by making use of the renormalization group equations.

\section{V. $n_{1} \rightarrow n_{2}$ PROCESSES.}

\section{A. Heuristic arguments.}

Let us consider the scattering of $n_{1}$ virtual particles of equal energies $\omega_{1}$ into $n_{2}$ virtual particles of equal energies $\omega_{2}$ in $\varphi^{4}$ theory. The amplitude of this process is given by the following path integral,

$$
\begin{aligned}
& A_{n_{1} \rightarrow n_{2}}= \\
& \int D \varphi \mathrm{e}^{i S}\left(\int \varphi(x, t) \mathrm{e}^{i \omega_{1} t} d \mathbf{x} d t\right)^{n_{1}}\left(\int \varphi(x, t) \mathrm{e}^{-i \omega_{2} t} d \mathbf{x} d t\right)^{n_{2}}
\end{aligned}
$$

Let us study the case when

$$
n_{1,2}=\frac{\nu_{1,2}}{\lambda}
$$

where $\nu_{1,2}$ are fixed as $\lambda \rightarrow 0$. The amplitude (5.1) can be written in the equivalent form,

$$
\begin{aligned}
& A_{n_{1} \rightarrow n_{2}}= \\
& \int D \varphi \exp \left(i S+n_{1} \ln \left(\int \varphi(x, t) \mathrm{e}^{i \omega_{1} t} d \mathbf{x} d t\right)+n_{2} \ln \left(\int \varphi(x, t) \mathrm{e}^{-i \omega_{2} t} d \mathbf{x} d t\right)\right)
\end{aligned}
$$

The change of variables

$$
\varphi=\frac{1}{\sqrt{\lambda}} \phi
$$

transforms the integral (5.2) into an apparently saddle point form

$$
\begin{aligned}
& A_{n_{1} \rightarrow n_{2}}= \\
& \lambda^{-\left(n_{1}+n_{2}\right) / 2} \int D \phi \exp \frac{1}{\lambda}\left(i S(\phi)+\nu_{1} \ln \left(\int \phi(x, t) \mathrm{e}^{i \omega_{1} t} d \mathbf{x} d t\right) \nu_{2} \ln \left(\int \phi(x, t) \mathrm{e}^{-i \omega_{2} t} d \mathbf{x} d t\right)\right)
\end{aligned}
$$

which indicates that the amplitude is exponential,

$$
A_{n_{1} \rightarrow n_{2}} \propto \lambda^{-\left(n_{1}+n_{2}\right) / 2} \exp \left(\frac{1}{\lambda} \Psi\left(\nu_{1}, \nu_{2}\right)\right)
$$


Equivalently, this expression can be written in a way conjectured in Introduction,

$$
A_{n_{1} \rightarrow n_{2}}=\sqrt{n_{1} !} \sqrt{n_{2} !} \mathrm{e}^{\frac{1}{\lambda} F\left(\nu_{1}, \nu_{2}\right)}
$$

where

$$
F\left(\nu_{1}, \nu_{2}\right)=\Psi\left(\nu_{1}, \nu_{2}\right)-\frac{\nu_{1}}{2} \ln \nu_{1}-\frac{\nu_{2}}{2} \ln \nu_{2}+\frac{\nu_{1}}{2}+\frac{\nu_{2}}{2}
$$

Similar observations can be made in the case when final and/or initial particles are on mass shell; a natural way to proceed in that case is to use the coherent state representation.

Note that the above observations do not take into account possible cancellations in the pre-exponential factor, which, in fact, do appear, at least at the tree level [29 31].

We have not been able to convert the above observations into a proof for the following reason. In general, eq.(5.1) contains disconnected graphs, so it does not, in fact, correspond to the connected amplitude. One may try to single out the connected amplitude by imposing a constraint that $n_{1}$ and $n_{2}$ are coprime numbers (up to a common divisor two). However, in that case the conservation of energy, $n_{1} \omega_{1}=n_{2} \omega_{2}$, would mean that $\omega_{1}$ and $\omega_{2}$ entering

eq.(5.3) are not arbitrary, but their ratio is a ratio of two large natural coprime numbers (the same is true for the ratio $\left.\nu_{1} / \nu_{2}\right)$. In this way large numbers enter the exponent in eq.(5.3) implicitly, in addition to explicit $1 / \lambda$. Thus, the saddle point nature of the integral (5.3) is questionable.

Nevertheless, we expect the functional form of the amplitude, eq. (5.4), to be correct. We check this conjecture by numerical calculations of the tree amplitudes in the next subsection.

\section{B. Numerical calculation of tree amplitudes.}

To construct the algorithm for numerical calculations of the tree amplitudes we make use of the following observation made in ref. [31].

Let us consider amplitudes of scattering of $n_{1}$ virtual particles into $n_{2}$ real particles when all particles are at rest. To avoid disconnected graphs, we keep $n_{1}$ and $n_{2}$ being coprime 
numbers up to one common divisor two. Let us construct an iteration solution to source-free classical space-independent field equation,

$$
\ddot{\varphi}+\varphi+\lambda \varphi^{3}=0
$$

(we again set the mass equal to one) with the first iteration

$$
\begin{gathered}
\phi^{(0)}=z_{1}+z_{2}, \\
z_{1}=\zeta_{1} \mathrm{e}^{i \omega_{1} t}, \quad z_{2}=\zeta_{2} \mathrm{e}^{-i \omega_{2} t},
\end{gathered}
$$

$\omega_{2}=1$ when we are interested in scattering into real particles. The iteration procedure is defined by the following equation

$$
\ddot{\varphi}^{(k)}+\varphi^{(k)}=-\lambda\left(\varphi^{3}\right)^{(k-1)}
$$

where $\left(\varphi^{3}\right)^{(k-1)}$ is of $(k-1)$-th order in $\lambda$ and is expressed through $\varphi^{(0)}, \ldots, \varphi^{(k-1)}$. At the $l$-th step $\left(l=\left(n_{1}+n_{2}\right) / 2-1\right)$, in addition to oscillating terms, a peculiar term appears for the first time, and the amplitude $A_{n_{1} \rightarrow n_{2}}$ is determined by its coefficient,

$$
\varphi^{(l)}=t \mathrm{e}^{-i t} \frac{A_{n_{1} \rightarrow n_{2}}(\omega)}{n_{1} !\left(n_{2}-1\right) !} \frac{1}{2} \zeta_{1}^{n_{1}} \zeta_{2}^{n_{2}-1}+\text { oscillating terms }
$$

This algorithm was adapted for computer calculations. When one expands $\varphi^{(i)}$ in powers of exponents $z_{1}$ and $z_{2}$, eq.(5.7) enables one to obtain the coefficients of the expansion of $\varphi^{(k)}$ algebraically. This induces an efficient numerical procedure for the calculation of the amplitude $A_{n_{1} \rightarrow n_{2}}$.

Since we are calculating only tree amplitudes, we can determine the power of $\lambda$ in the resulting amplitudes directly, by counting the number of vertices in the diagrams,

$$
A_{n_{1} \rightarrow n_{2}}^{\text {tree }} \propto \lambda^{\frac{n_{1}+n_{2}}{2}-1}
$$

By comparing eqs.(5.4) and (5.9), one finds that the function $F$ should be a homogeneous function of the first order, so it should have the following form, 


$$
F^{\text {tree }}\left(\lambda n_{1}, \lambda n_{2}\right)=\lambda\left(n_{1}+n_{2}\right) \Phi(\nu)
$$

where $\nu=n_{2} / n_{1}$, and the entire tree amplitude can be rewritten as follows,

$$
A_{n_{1} \rightarrow n_{2}}^{\text {tree }}=\left(n_{1}-1\right) !\left(n_{2}-1\right) ! \lambda^{\frac{n_{1}+n_{2}}{2}-1} \mathrm{e}^{\left(n_{1}+n_{2}\right) \Phi\left(n_{2} / n_{1}\right)+O\left(n^{0}\right)}
$$

So, to check our conjecture at the tree level, we calculate the function

$$
\Omega\left(n_{1}, n_{2}\right)=\log \frac{A_{n_{1} \rightarrow n_{2}}^{\text {tree }}}{\left(n_{1}-1\right) !\left(n_{2}-1\right) !}
$$

and verify that in the large $-n$ limit, this function $\Omega$ is linear at $n_{1}$ at given $\nu$. So, we should calculate $\Omega$ at different $n_{1}$ and at fixed $\nu$ and then observe that at large $n_{1}$ it tends to a linear function at each $\nu$.

In real calculations, we cannot fix $\nu$ exactly: if we choose a particular $n_{1}$, then at given $\nu$ the "number of outgoing particles", $\nu n_{1}$, would not be, in general, integer. So, for fixed $\nu$, as we vary $n_{1}$, we can only choose an integer $n_{2}$ in such a way that $n_{2} / n_{1}$ is close to $\nu$, and $n_{2}$ is coprime to $n_{1}$; the larger $n_{1}$, the closer $n_{2} / n_{1}$ to $\nu$. To have better precision, for each $n_{1}$ we have used three values of $n_{2}$ giving $n_{2} / n_{1}$ closest to $\nu$ (two from above and one from below) and then made the interpolation to the chosen value of $\nu$. The functions $\Omega_{\nu}\left(n_{1}\right)$, obtained in this way, are shown in fig. 10 (unbroken theory) and fig. 11 (broken theory).

Figs. 10 and 11 are consistent with $\Omega_{\nu}\left(n_{1}\right)$ being linear function of $n_{1}$ at large $n_{1}$ at various $\nu$. This means that the function $\Phi\left(n_{1}, n_{2}\right)$ entering eq.(5.10) indeed depends only on $n_{2} / n_{1}$, i.e., our conjecture (5.4) is valid at the tree level.

\section{CONCLUSIONS}

In complete analogy to the instanton-like processes in high energy collisions, there emerges an intriguing situation with calculations of the amplitudes of processes with large number of outgoing particles $(n=O(1 / \lambda))$ in scalar theories. The results of this paper strongly suggest that the corresponding cross section is of the exponential form,

$$
\sigma_{n} \propto \mathrm{e}^{\frac{1}{\lambda} G(\lambda n, \lambda E)}
$$


where $E$ is the total center-of-mass energy. This form indicates that the exponent $G$ may be calculable in a semiclassical manner, but the actual calculational technique is presently lacking. The perturbative calculations can at best provide the evaluation of first terms in the expansion of $G$ in $(\lambda n)$. This expansion blows up at $(\lambda n) \sim 1$, so the perturbation theory is of no use for studying the most interesting values of $n$.

In the instanton-like case, several proposals have been put forward which might enable one to calculate the exponent for the cross section. These include the Landau technique for the calculation of the semiclassical matrix elements [16,17 and the study of multiparticle initial states [12 14]. If either of these approaches works for multiparticle production in scalar theories, the exponent in eq.(6.1) should be independent of a particular choice of the initial state (whether it contains one or several particles, whether these particles are virtual or real, etc.). We hope that this expectation can be checked by making use of the techniques developed in this paper.

It might happen that the exponent $G$ in eq. (6.1) is negative at all $n$ and $E$, so that the multiparticle cross sections are always exponentially small. Even in that case the calculation of the exponent would be of substantial interest as it would require the development of novel methods of quantum field theory.

The authors are indebted to M.B.Voloshin for pointing out the peculiar infrared properties of scalar theories in $(2+1)$ dimensions, for numerous useful discussions and help in computer calculations. We thank C.Bachas, H.Goldberg, A.Kuznetsov, Yu.Makeenko, E.Mottola, A.Ringwald, P.Tinyakov and L.Yaffe for helpful discussions.

This work is supported in part by International Science Foundation, grant \# MKT 000. The work of D.T.S. is supported in part by Russian Foundation for Fundamental Research, grant \# 93-02-3812. 


\section{REFERENCES}

[1] A. Ringwald, Nulc. Phys. B330, 1 (1990).

[2] O. Espinosa, Nulc. Phys. B343, 310 (1990).

[3] M. P. Mattis, Phys. Rep. 214, 159 (1992).

[4] P. G. Tinyakov, Int. J. Mod. Phys. A8, 1823 (1993).

[5] J. M. Cornwall, Phys. Lett. B243, 271 (1990).

[6] H. Goldberg, Phys. Lett. B246, 445 (1990).

[7] M. B. Voloshin, Nucl. Phys. B383, 233 (1992).

[8] S. Yu. Khlebnikov, V. A. Rubakov and P. G. Tinyakov, Nucl. Phys. B350, 441 (1991).

[9] P. B. Arnold and M. P. Mattis, Phys. Rev. D42, 1738 (1990).

[10] L. G. Yaffe, "Scattering amplitudes in instanton background", in Proc. Santa Fe Workshop, eds. M.Mattis and E.Mottola (World Scientific, Singapore, 1990).

[11] A. Mueller, Nucl. Phys. B353, 44 (1991); Xu Li, L. McLerran, M. B. Voloshin and Rong-tai Wang, Phys. Rev. D44, 2899 (1991).

[12] V. A. Rubakov and P. G. Tinyakov, Phys. Lett. B279, 165 (1992).

[13] P. G. Tinyakov, Phys. Lett. B284, 410 (1992).

[14] V. A. Rubakov, D. T. Son and P. G. Tinyakov, Phys. Lett. B287, 342 (1992).

[15] M. P. Mattis, L. McLerran and L. G. Yaffe, Phys. Rev. D45, 4294 (1992).

[16] S. Yu. Khlebnikov, Phys. Lett. B282, 459 (1992).

[17] D. I. Diakonov and V. Yu. Petrov, Phys. Rev. D50, 266 (1994).

[18] V. I. Zakharov, Nucl. Phys. B383, 218 (1992). 
[19] L. S. Brown, Phys. Rev. D46, 4125 (1992).

[20] E. N. Argyres, R. H. P. Kleiss and C. G. Papadopoulos, Nucl. Phys. B391, 42 (1993).

[21] M. V. Libanov, V. A. Rubakov and S. V. Troitsky, Nucl. Phys. B412, 607 (1994).

[22] M. B. Voloshin, Phys. Rev. D47, 357 (1993).

[23] B. H. Smith, Phys. Rev. D47, 3518 (1993).

[24] E. N. Argyres, R. H. P. Kleiss and C. G. Papadopoulos, Phys. Lett. B308, 292 (1993).

[25] Yu. Makeenko, Exact Multiparticle Amplitude at Threshold in Large $-N$ Component $\phi^{4}$ Theory, Niels Bohr Inst. preprint NBI-HE-94-25 (1994).

[26] V. A. Rubakov and D. T. Son, Renormalization group for multiparticle production in $(2+1)$ dimensions around the threshold, hep-ph/9406362, Proc. Int. Seminar Quarks-94, 1994, to appear.

[27] C. Thorn, Phys. Rev. D6, 39 (1979); see also K. Huang, Quarks, Leptons and Gauge Fields, $§ 10.8$ (World Scientific, 1982).

[28] A. S. Gorsky and M. B. Voloshin, Phys. Rev. D48, 3843 (1993).

[29] M. B. Voloshin, Phys. Rev. D47, 2573 (1993).

[30] E. N. Argyres, R. H. P. Kleiss and C. G. Papadopoulos, Phys. Lett. B308, 315 (1993).

[31] M. V. Libanov, V. A. Rubakov and S. V. Troitsky, Phys. Lett. B318, 134 (1993). 


\section{FIGURES}

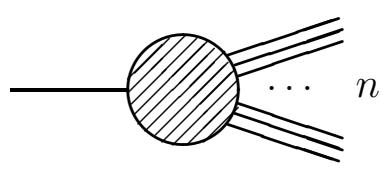

FIG. 1. Process "few $\rightarrow$ many"

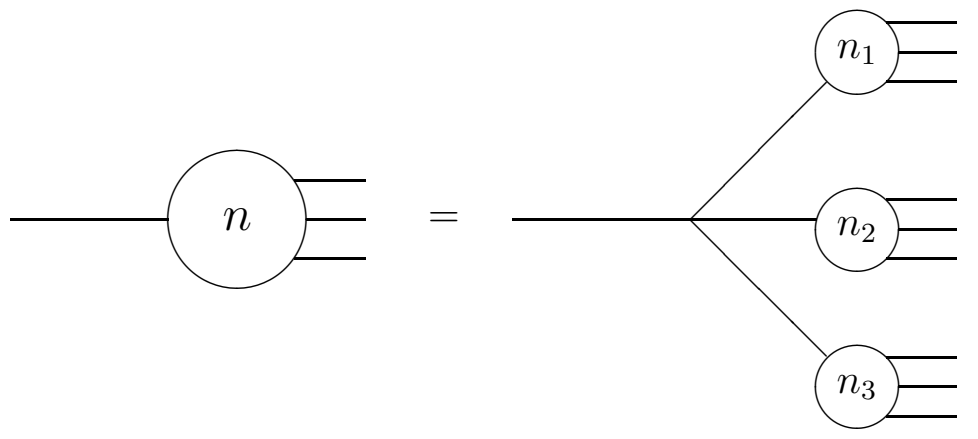

FIG. 2. Recurrence relation for tree amplitudes

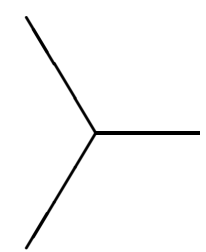

$-6 \lambda \varphi_{0}$

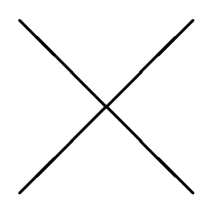

$-6 \lambda$

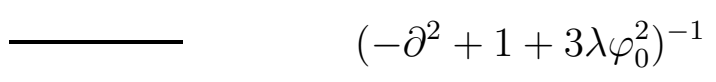

FIG. 3. Feynman rules for calculating multiparticle amplitudes 


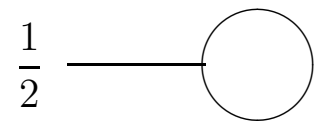

a

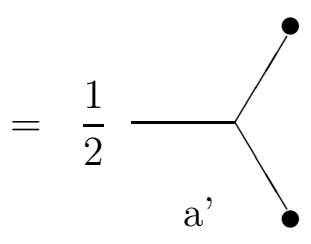

FIG. 4. The one-loop graph (a) and a representation (a') of its leading singularity
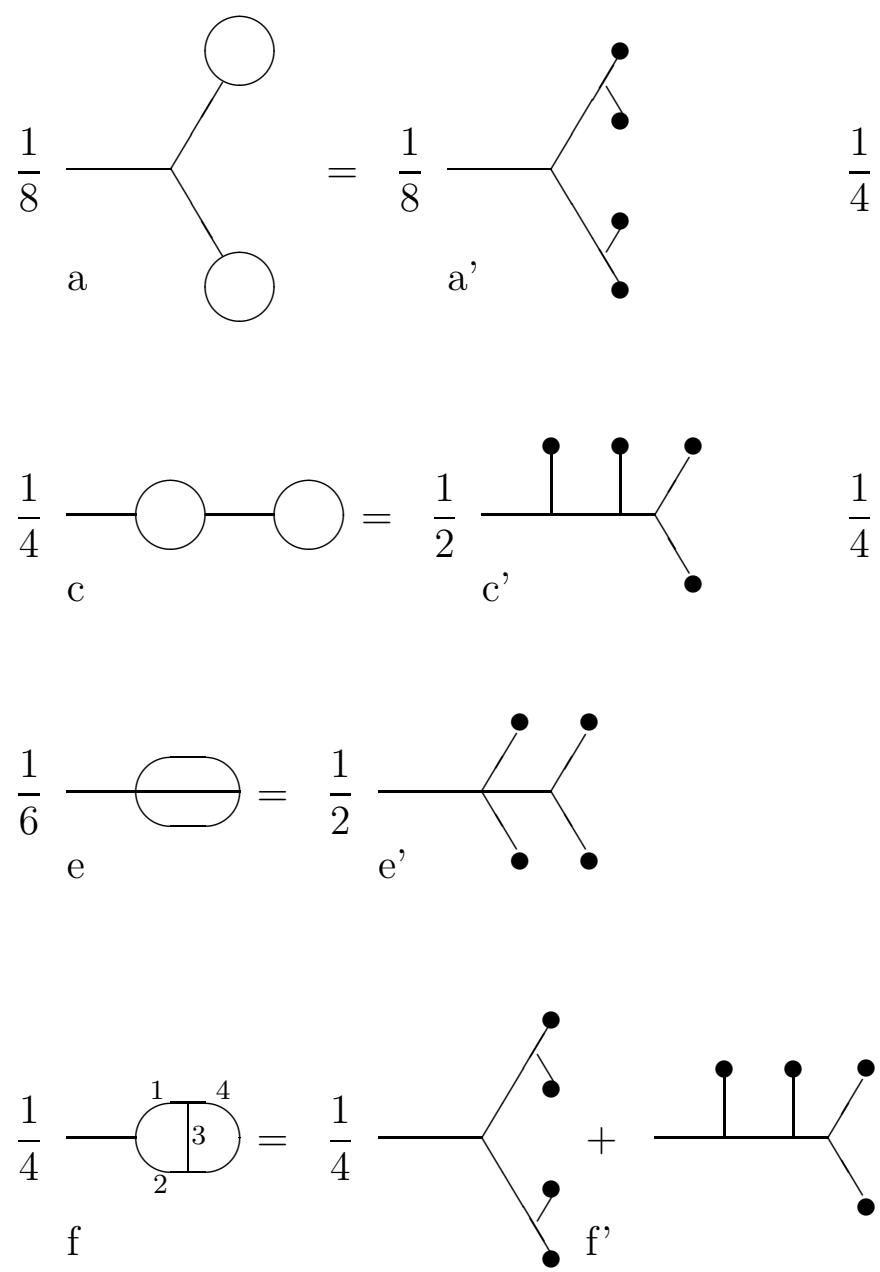

FIG. 5. The two-loop graphs with their symmetry factors (a-f) and their leading singularities $\left(a^{\prime}-f^{\prime}\right)$ 


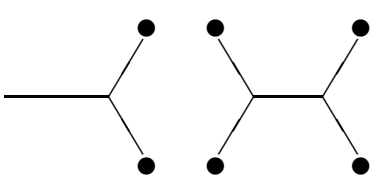

FIG. 6. A disconnected graph giving subleading contribution at large $n$.

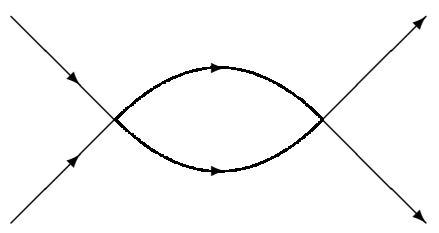

FIG. 7. The graph contributing to the lowest-order beta function in the effective non-relativistic theory. 

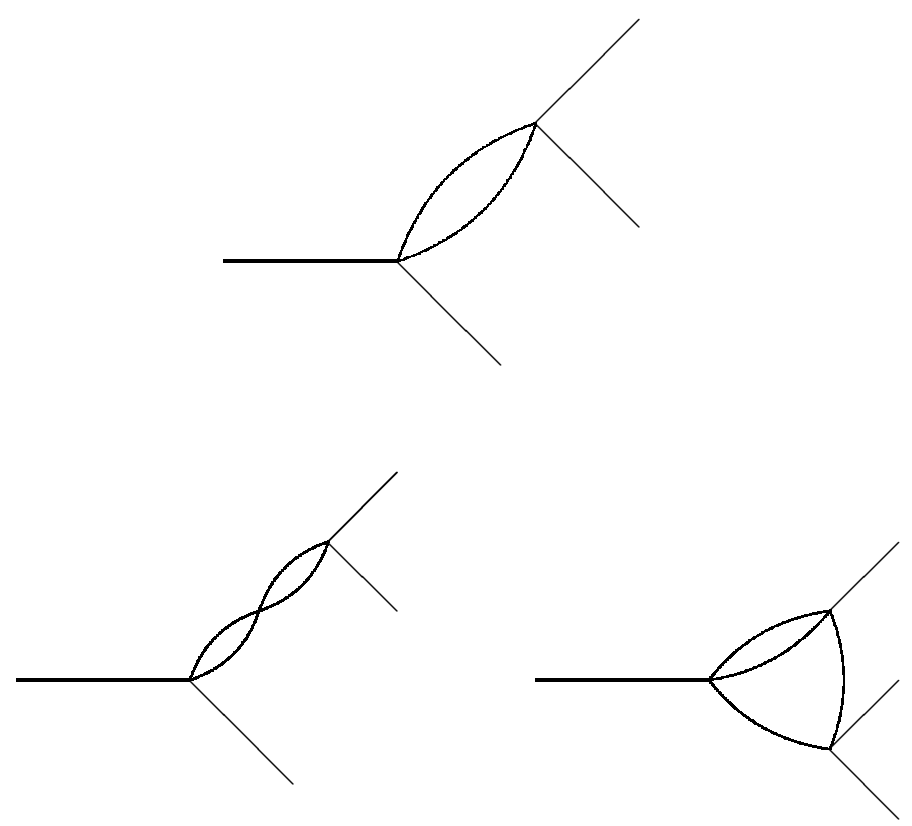

FIG. 8. One- and two-loop graphs that contribute to the $1 \rightarrow 3$ amplitude in the infrared

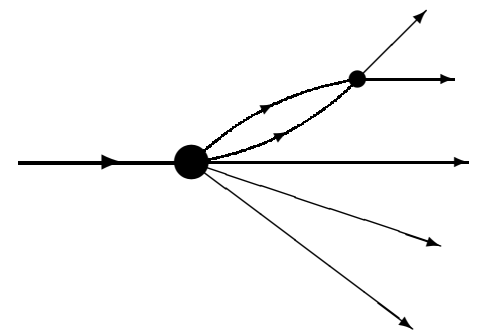

FIG. 9. A graph that makes contribution to RG equation for $1 \rightarrow n$ amplitude. There are $n(n-1) / 2$ diagrams of this type, corresponding to different possiblities to choose a pair of final particles to rescatter.

FIG. 10. Numerical results for $n_{1} \rightarrow n_{2}$ tree amplitudes in the unbroken $\varphi^{4}$ theory (see Postscript file)

FIG. 11. Numerical results for $n_{1} \rightarrow n_{2}$ tree amplitudes in the broken $\varphi^{4}$ theory (see Postscript file) 